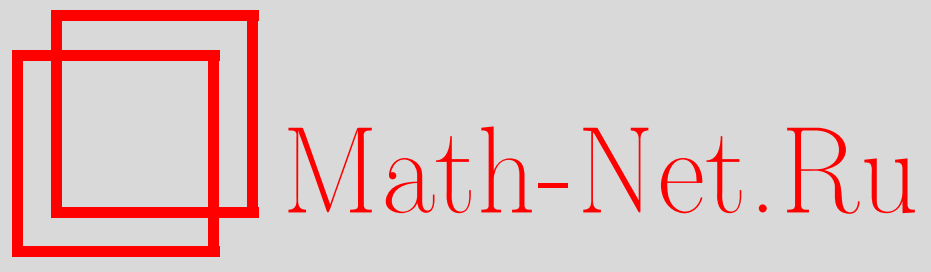

М. О. Катанаев, Полиномиальная гамильтонова форма общей теории относительности, ТМФ, 2006, том 148, номер 3, 459-494

DOI: https://doi.org/10.4213/tmf2327

Использование Общероссийского математического портала Math-Net.Ru подразумевает, что вы прочитали и согласны с пользовательским соглашением http://www . mathnet.ru/rus/agreement

Параметры загрузки:

IP: 3.91 .87 .62

26 апреля 2023 г., 16:35:40

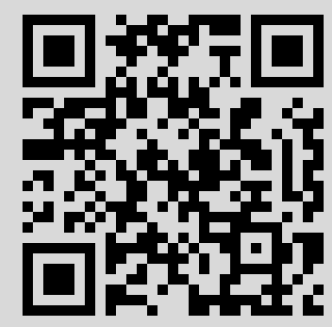




\title{
ПОЛИНОМИАЛЬНАЯ ГАМИЛЬТОНОВА ФОРМА ОБЩЕЙ ТЕОРИИ ОТНОСИТЕЛЬНОСТИ
}

\begin{abstract}
Фазовое пространство общей теории относительности расширено до пуассонова многообразия путем включения определителя метрики и сопряженного импульса в качестве дополнительных независимых переменных. В результате действие и связи принимают полиномиальный вид. Предложено выражение для производящего функционала функций Грина. Показано, что скобка Дирака определяет вырожденную пуассонову структуру на многообразии, а связи второго рода являются функциями Казимира относительно этой структуры. В качестве примера использования новых переменных рассмотрена вселенная Фридмана.
\end{abstract}

Ключевые слова: общая теория относительности, гамильтонов формализм.

\section{1. ВВЕДЕНИЕ}

Каноническая формулировка любой модели математической физики представляет собой важнейший этап исследования уравнений движения, в частности при постановке и анализе задачи Коши. Она является также основой для канонического квантования моделей.

Каноническая формулировка общей теории относительности (ОТО) технически сложна, и ей посвящено много работ. Отметим только некоторые из них. Самосогласованная формулировка ОТО на гамильтоновом языке впервые была дана Дираком в формализме второго порядка [1]. Он рассматривал в качестве независимых переменных компоненты метрики $g_{\alpha \beta}$ и показал, что гамильтониан гравитационного поля равен линейной комбинации связей. Затем Арновитт, Дезер и Мизнер в серии статей, завершившихся обзором [2], существенно упростили вычисления и прояснили геометрический смысл канонических импульсов, выразив их через внешнюю кривизну пространственной гиперповерхности, вложенной в четырехмерное пространствовремя. Выражение для гамильтониана было найдено ими в формализме первого порядка, когда метрика $g_{\alpha \beta}$ и симметричная аффинная связность $\Gamma_{\{\alpha \beta\}}{ }^{\gamma}$ рассматриваются в качестве независимых переменных. По сути дела этот подход и упростил вычисления. В пионерских работах [1], [2] не была посчитана явно алгебра связей,

* Математический институт им. В. А. Стеклова, Москва, Россия. E-mail: katanaev@mi.ras.ru 
но показана их согласованность с уравнениями движения (связи первого рода). В статье [2] была также проанализирована роль граничных членов и дано определение полной энергии гравитационного поля в виде поверхностного интеграла для асимптотически плоского пространства-времени. Роль граничных слагаемых в ОТО была более полно рассмотрена в работе [3].

В статье [4] дана каноническая формулировка ОТО и подробно рассмотрено обобщение уравнения Шредингера для волновой функции вселенной, которое в дальнейшем получило название уравнения Уилера-де Витта. Здесь впервые явно вычислена алгебра связей ОТО.

В статье [5] получена алгебра связей для произвольной модели, инвариантной относительно общих преобразований координат, при предположениях, что теория самосогласована и связи генерируют общие преобразования координат для канонических переменных (см. также работу [6]). Отметим, что предположение о самосогласованности модели (замкнутости алгебры связей) является слишком сильным. Поскольку заранее это не известно, то, наоборот, для заданной модели необходимо явно вычислить алгебру связей, чтобы доказать самосогласованность теории.

Исследование гамильтоновой формулировки ОТО в реперном формализме было начато Дираком [7] и Швингером [8], которые для простоты использовали временну́ю калибровку. Гамильтонов анализ в общем случае, без фиксирования калибровки, был проведен значительно позже из-за значительных технических трудностей [9], [10].

Гамильтонова динамика ОТО содержит связи, которые неполиномиальны по компонентам метрики пространственного сечения, что является существенной трудностью для анализа и квантования теории гравитации. В последние годы большое внимание привлекла полиномиальная гамильтонова формулировка, данная Аштекаром [11]. Он предложил использовать комплексные координаты в расширенном фазовом пространстве, которые являются тензорными плотностями и приводят к полиномиальным связям. В настоящей статье рассмотрен другой способ расширения вещественного фазового пространства [12], когда в качестве дополнительных координат выбираются определитель метрики и сопряженный импульс. Показано, что пуассонова структура на расширенном пространстве является вырожденной, а исходное фазовое пространство отображается на подмногообразие расширенного пуассонова многообразия с помощью канонического преобразования. Все новые канонические переменные вещественны, являются тензорными плотностями, а связи принимают полиномиальный вид.

Предложено также новое выражение для производящего функционала функций Грина в виде функционального интеграла по пуассонову многообразию. Эта форма интеграла сводится к стандартному выражению для производящего функционала в фазовом пространстве [13] после интегрирования по дополнительным переменным, которое снимается двумя дополнительными $\delta$-функциями. Доказано, что соответствующий якобиан преобразования координат равен единице.

План статьи следующий. В разделах 2-6 подробно описан переход от лагранжиана Гильберта-Эйнштейна к гамильтониану. Это сделано для того, чтобы не отсылать читателя к первоисточникам, в которых значительная часть вычислений 
обычно опускается. Кроме того, при описании геометрии гиперповерхностей в разделе 3 рассмотрен общий случай аффинной геометрии, заданной в пространствевремени. При этом антисимметричная часть внешней кривизны гиперповерхности определяется тензором кручения. Это важно для канонической формулировки ОТО в реперном формализме и для гамильтоновой формулировки моделей абсолютного параллелизма. В разделе 7 описано каноническое преобразование между фазовым пространством ОТО и подмногообразием расширенного пуассонова многообразия. Показано, что в расширенном пространстве все связи и действие модели принимают полиномиальный вид, а также вычислена алгебра связей. В разделе 8 предложено выражение для производящего функционала функций Грина на пуассоновом многообразии. В качестве приложения новых переменных в заключительном разделе рассмотрен случай однородной и изотропной вселенной, в котором все вычисления можно легко проверить.

\section{2. АДМ-ПАРАМЕТРИЗАЦИЯ МЕТРИКИ}

При анализе гамильтоновой структуры уравнений ОТО Арновитт, Дезер и Мизнер [2] использовали специальную параметризацию (АДМ-параметризацию) метрики, которая существенно упростила вычисления.

Рассмотрим многообразие $\mathbb{M}, \operatorname{dim} \mathbb{M}=n$, с метрикой лоренцевой сигнатуры $(+-\cdots-)$. Мы намеренно не ограничиваемся наиболее важным случаем четырехмерного пространства-времени, так как модели гравитации в меньшем и большем числе измерений в последнее время привлекают большое внимание. Пусть $\left\{x^{\alpha}\right\}$, $\alpha=0,1, \ldots, n-1,-$ система локальных координат. Выделим среди координат время $t=x^{0}$, тогда $\left\{x^{\alpha}\right\}=\left\{x^{0}, x^{\mu}\right\}, \mu=1, \ldots, n-1$. В дальнейшем буквы из начала греческого алфавита $(\alpha, \beta, \ldots)$ будут пробегать все значения индексов, а из середины $(\mu, \nu, \ldots)$ - только пространственные значения. Это правило легко запомнить по следующим включениям: $\{\mu, \nu, \ldots\} \subset\{\alpha, \beta, \ldots\}$ и $\{1,2, \ldots\} \subset\{0,1,2, \ldots\}$. АДМпараметризация произвольной метрики имеет вид

$$
g_{\alpha \beta}=\left(\begin{array}{cc}
N^{2}+N^{\rho} N_{\rho} & N_{\nu} \\
N_{\mu} & g_{\mu \nu}
\end{array}\right),
$$

где $g_{\mu \nu}$ - метрика на $(n-1)$-мерных сечениях многообразия $x^{0}=$ const. В выбранной параметризации вместо $n$ компонент метрики, содержащих хотя бы один временно́й индекс, $g_{00}$ и $g_{0 \mu}$, введено такое же число функций $N$ и $N_{\mu}$. Выше $N^{\rho}=\hat{g}^{\rho \mu} N_{\mu}$, где $\hat{g}^{\rho \mu}-(n-1) \times(n-1)$-матрица, обратная к $g_{\mu \nu}$ :

$$
\hat{g}^{\rho \mu} g_{\mu \nu}=\delta_{\nu}^{\rho},
$$

которую мы называем обратной метрикой на сечениях $x^{0}=$ const. В дальнейшем подъем пространственных индексов будет всегда осуществляться с помощью обратной метрики $\hat{g}^{\rho \mu}$, помеченной шляпкой и не совпадающей с пространственной частью метрики $g^{\alpha \beta}$, обратной к $g_{\alpha \beta}, \quad \hat{g}^{\rho \mu} \neq g^{\rho \mu}$. Функция $N=N(x)$ называется функцией хода, а функции $N_{\mu}=N_{\mu}(x)$ - функциями сдвига. Не ограничивая общности, можно считать, что функция хода положительна $(N>0)$. В этом случае АДМ-параметризация метрики (1) является взаимно однозначной. 
Интервал, соответствующий параметризации (1), имеет вид

$$
d s^{2}=N^{2} d t^{2}+g_{\mu \nu}\left(d x^{\mu}+N^{\mu} d t\right)\left(d x^{\nu}+N^{\nu} d t\right) .
$$

Мы предполагаем, что координата $x^{0}=t$ является временем, т.е. касательный вектор $\partial_{0}$ к координатной линии $x^{0}$ времениподобен. Формально это условие записывается в виде

$$
\left(\partial_{0}, \partial_{0}\right)=g_{00}=N^{2}+N^{\rho} N_{\rho}>0 .
$$

В этом случае метрика $g_{\alpha \beta}$ имеет лоренцеву сигнатуру тогда и только тогда, когда матрица

$$
g_{\mu \nu}-\frac{N_{\mu} N_{\nu}}{N^{2}+N^{\rho} N_{\rho}}
$$

отрицательно определена. Отметим, что сама метрика $g_{\mu \nu}$, индуцированная на сечениях $x^{0}=$ const, может и не быть отрицательно определенной. Это значит, что сечение $x^{0}=$ const в общем случае не является пространственноподобным. В дальнейшем мы будем дополнительно предполагать, что координаты выбраны таким образом, чтобы все сечения $x^{0}=$ const были пространственноподобны, т.е. метрика $g_{\mu \nu}$ также отрицательно определена. Это удобно для постановки задачи Коши, когда начальные данные задаются на пространственноподобной поверхности и рассматривается их эволюция во времени.

Аналогичным образом можно параметризовать метрику и на римановом многообразии с положительно определенной метрикой, для этого вместо времени достаточно явно выделить произвольную координату.

Метрика, обратная к (1), имеет вид

$$
g^{\alpha \beta}=\left(\begin{array}{cc}
\frac{1}{N^{2}} & -\frac{N^{\nu}}{N^{2}} \\
-\frac{N^{\mu}}{N^{2}} & \hat{g}^{\mu \nu}+\frac{N^{\mu} N^{\nu}}{N^{2}}
\end{array}\right) .
$$

Пространственная матрица в правом нижнем блоке

$$
g^{\mu \nu}=\hat{g}^{\mu \nu}+\frac{N^{\mu} N^{\nu}}{N^{2}},
$$

как легко проверить, является обратной к метрике (3). Это значит, что отрицательная определенность метрики (3) эквивалентна отрицательной определенности матрицы $g^{\mu \nu}$.

Заметим, что если метрика на многообразии $\mathbb{M}$ имеет лоренцеву сигнатуру, то условие пространственноподобности всех сечений $x^{0}=$ const эквивалентно условию $N^{2}>0$. Действительно, из отрицательной определенности $g_{\mu \nu}$ следует отрицательная определенность обратной матрицы $\hat{g}^{\mu \nu}$. Тогда из уравнения (5) вытекает отрицательная определенность матрицы

$$
g^{\mu \nu}-\frac{N^{\mu} N^{\nu}}{N^{2}}
$$

Это, в свою очередь, эквивалентно условию $g^{00}>0$ или $N^{2}>0$.

Чтобы продемонстрировать тонкости, которые могут возникнуть при АДМ-параметризации метрики, приведем простой пример. 


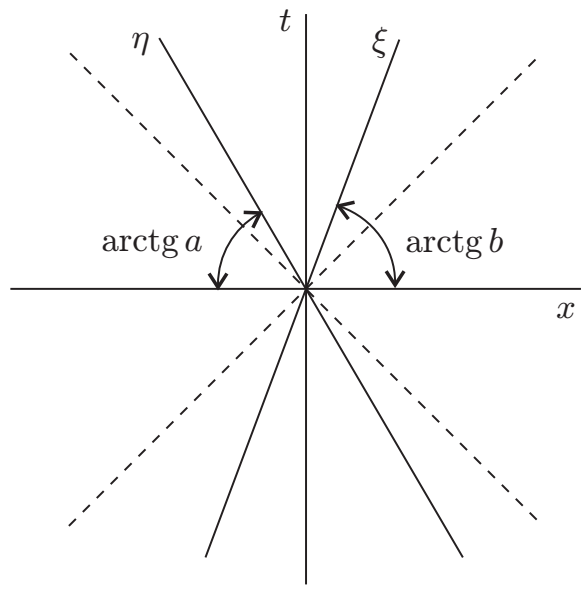

ПримеР. Рассмотрим двумерное пространство-время Минковского $\mathbb{R}^{1,1}$ с декартовыми координатами $t, x$. Введем новую систему координат $\xi, \eta$, зависящую от двух вещественных параметров $a$ и $b$ (см. рисунок):

$$
\xi=t+a x, \quad \eta=t-b x, \quad|a| \neq 1, \quad|b| \neq 1, \quad a+b \neq 0 .
$$

Легко получить формулы обратного преобразования

$$
t=\frac{b \xi+a \eta}{a+b}, \quad x=\frac{\xi-\eta}{a+b} .
$$

В новых координатах лоренцева метрика имеет вид

$$
d s^{2}=d t^{2}-d x^{2}=\frac{1}{(a+b)^{2}}\left[\left(b^{2}-1\right) d \xi^{2}+2(a b+1) d \xi d \eta+\left(a^{2}-1\right) d \eta^{2}\right] .
$$

Проанализируем АДМ-параметризацию метрики в координатах $x^{0}=\xi, x^{1}=\eta$

$$
g_{00}=\frac{b^{2}-1}{(a+b)^{2}}, \quad g_{01}=\frac{a b+1}{(a+b)^{2}}, \quad g_{11}=\frac{a^{2}-1}{(a+b)^{2}} .
$$

Функции хода и сдвига имеют вид

$$
N^{2}=-\frac{1}{a^{2}-1}, \quad N_{1}=\frac{a b+1}{(a+b)^{2}} .
$$

Из условий $g_{00}>0$ и $g_{11}<0$ следуют соответственно неравенства $|b|>1$ и $|a|<1$. Мы видим, что эти условия являются необходимыми и достаточными для того, чтобы координатная линия $\xi$ была времениподобной, а $\eta$ - пространственноподобной. Нетрудно также проверить эквивалентность условий

$$
\begin{aligned}
& g_{00}>0 \quad \Leftrightarrow \quad g_{11}-\frac{N_{1} N_{1}}{N^{2}+N^{1} N_{1}}=-\frac{1}{b^{2}-1}<0, \\
& g^{00}>0 \quad \Leftrightarrow \quad \hat{g}^{11}=g^{11}-\frac{N^{1} N^{1}}{N^{2}}=\frac{(a+b)^{2}}{a^{2}-1}<0 .
\end{aligned}
$$


Используя формулу для определителя блочных матриц, получаем выражение для определителя метрики (1):

$$
\operatorname{det} g_{\alpha \beta}=N^{2} \operatorname{det} g_{\mu \nu} .
$$

Отсюда следует выражение для элемента объема

$$
e=N \hat{e}, \quad e=\sqrt{\left|\operatorname{det} g_{\alpha \beta}\right|}, \quad \hat{e}=\sqrt{\left|\operatorname{det} g_{\mu \nu}\right|} .
$$

Последняя формула является обобщением хорошо известного из школьного курса правила: объем призмы равен произведению площади основания на высоту. В рассматриваемом случае площадью основания является $\hat{e}$, а высотой - функция хода $N$.

При проведении вычислений оказываются полезными следующие формулы, которые проверяются прямо:

$$
\begin{gathered}
g^{00} g^{\mu \nu}-g^{0 \mu} g^{0 \nu}=\frac{\hat{g}^{\mu \nu}}{N^{2}} \\
g^{\sigma \mu} g^{0 \nu}-g^{\sigma \nu} g^{0 \mu}=\frac{N^{\mu} \hat{g}^{\sigma \nu}-N^{\nu} \hat{g}^{\sigma \mu}}{N^{2}} \\
g^{\mu \nu} g_{\nu \sigma}=\delta_{\sigma}^{\mu}+\frac{N^{\mu} N_{\sigma}}{N^{2}} \\
g^{\mu \nu} g_{\mu \nu}=n-1+\frac{N^{\mu} N_{\mu}}{N^{2}}
\end{gathered}
$$

\section{3. ГЕОМЕТРИЯ ГИПЕРПОВЕРХНОСТЕЙ}

При гамильтоновой формулировке моделей гравитации мы рассматриваем пространство-время как семейство пространственных гиперповерхностей $x^{0}=$ const, которое параметризуется временем. Другими словами, для каждого момента времени пространство представляет собой гиперповерхность, вложенную в пространствовремя. Поскольку уравнения моделей гравитации определяют геометрию всего пространства-времени, то полезно знать, какая геометрия возникает при этом на пространственных сечениях. В настоящем разделе мы подойдем к этому вопросу с общей точки зрения, предполагая, что на объемлющем многообразии задана произвольная аффинная геометрия, и не предполагая лоренцевой сигнатуры метрики.

Рассмотрим $(n-1)$-мерную гиперповерхность $\mathbb{U}$, вложенную в $n$-мерное многообразие $\mathbb{M}$ :

$$
f: \mathbb{U} \rightarrow \mathbb{M}
$$

Обозначим координаты на $\mathbb{M}$ и $\mathbb{U}$ соответственно через $x^{\alpha}, \quad \alpha=0,1, \ldots, n-1$, и $u^{i}, \quad i=1, \ldots, n-1$. Тогда вложение $\mathbb{U}$ в $\mathbb{M}$ локально задается $n$ функциями $x^{\alpha}(u)$, которые предполагаются достаточно гладкими. Произвольное векторное поле $\left\{X^{i}\right\} \in \mathbb{T}(\mathbb{U})$ на гиперповерхности отображается в векторное поле $\left\{X^{\alpha}\right\} \in \mathbb{T}(\mathbb{M})$ на $\mathbb{M}$ с помощью дифференциала отображения

$$
f_{*}: X=X^{i} \partial_{i} \in \mathbb{T}(\mathbb{U}) \rightarrow Y=Y^{\alpha} \partial_{\alpha} \in \mathbb{T}(\mathbb{M}),
$$

где

$$
Y^{\alpha}=e^{\alpha}{ }_{i} X^{i}, \quad e^{\alpha}{ }_{i}=\partial_{i} x^{\alpha} .
$$


Матрица Якоби $e^{\alpha}{ }_{i}$ преобразования $f$ прямоугольна, имеет размер $n \times(n-1)$, ранг $n-1$ и, естественно, необратима. Она определена не на всем многообразии $\mathbb{M}$, а только на поверхности $\mathbb{U}$. Отметим также, что матрица Якоби является вектором и ковектором относительно преобразований координат на $\mathbb{M}$ и $\mathbb{U}$, соответственно Возврат отображения $f$ каждому ковекторному полю на образе $f(\mathbb{U}) \subset \mathbb{M}$ ставит в соответствие ковекторное поле на $\mathbb{U}$ :

$$
f^{*}: A=d x^{\alpha} A_{\alpha} \in \mathbb{T}^{*}(\mathbb{M}) \rightarrow B=d u^{i} B_{i} \in \mathbb{T}^{*}(\mathbb{U}),
$$

где

$$
B_{i}=A_{\alpha} e^{\alpha}{ }_{i}
$$

В дальнейшем мы будем отождествлять гиперповерхность $\mathbb{U}$ с ее образом $\mathbb{U}=$ $f(\mathbb{U}) \subset \mathbb{M}$.

1-Форма $n=d x^{\alpha} n_{\alpha}$, определяемая на гиперповерхности $\mathbb{U}$ системой алгебраических уравнений

$$
n_{\alpha} e^{\alpha}{ }_{i}=0, \quad i=1, \ldots, n-1,
$$

задает в касательном расслоении $\mathbb{T}(\mathbb{M})$ поле $(n-1)$-мерных подпространств, касательных к $\mathbb{U}$. Эти уравнения имеют единственное решение с точностью до умножения на произвольную отличную от нуля функцию, поскольку из определения вложения следует, что ранг матрицы Якоби равен $n-1$.

Матрица Якоби $e^{\alpha}{ }_{i}$ задает в касательных пространствах $\mathbb{T}_{x}(\mathbb{M}), x \in \mathbb{U}$, набор из $n-1$ векторов $e_{i}=e^{\alpha}{ }_{i} \partial_{\alpha}$, которые образуют базис касательного пространства $\mathrm{k}$ гиперповерхности.

Это все, что можно сказать о гиперповерхности $\mathbb{U}$, если задано только вложение (9). Теория становится намного более содержательной, если на многообразии $\mathbb{M}$ заданы дополнительные структуры. Остановимся на этом вопросе подробно.

Пусть на $\mathbb{M}$ задана аффинная геометрия, т.е. метрика $g_{\alpha \beta}$ и связность $\Gamma_{\alpha \beta}{ }^{\gamma}$. Рассмотрим, какая геометрия возникает на гиперповерхности $\mathbb{U} \subset \mathbb{M}$. Возврат отображения $f^{*}$ индуцирует на гиперповерхности единственную метрику

$$
f^{*}: g_{\alpha \beta} \rightarrow g_{i j}=g_{\alpha \beta} e^{\alpha}{ }_{i} e^{\beta}{ }_{j} .
$$

Наличие метрик $g_{\alpha \beta}$ и $g_{i j}$ соответственно на $\mathbb{M}$ и $\mathbb{U}$ позволяет опускать и поднимать индексы у матрицы Якоби

$$
e_{\alpha}{ }^{i}=g_{\alpha \beta} e^{\beta}{ }_{j} g^{i j},
$$

где $g^{i j}$ - метрика, обратная к $g_{i j}$. Эта матрица проектирует произвольный вектор из $\mathbb{T}_{x}(\mathbb{M}), x \in \mathbb{U}$, в касательное пространство к гиперповерхности $\mathbb{T}(\mathbb{U})$ :

$$
X^{\alpha} \rightarrow X^{i}=X^{\alpha} e_{\alpha}^{i}
$$

Теперь определим связность на гиперповерхности $\mathbb{U} \subset \mathbb{M}$ с помощью соотношения

$$
\widehat{\nabla}_{i} X^{k}=\left(\nabla_{\alpha} X^{\beta}\right) e^{\alpha}{ }_{i} e_{\beta}{ }^{k} .
$$

5 Теоретическая и математическая физика, т. 148, № 3, 2006 г. 
Раскрытие этого равенства приводит к выражению для индуцированной связности на гиперповерхности $\mathbb{U}$ в координатах

$$
\widehat{\Gamma}_{i j}{ }^{k}=\left(\partial_{i j}^{2} x^{\gamma}+\Gamma_{\alpha \beta}{ }^{\gamma} e^{\alpha}{ }_{i} e^{\beta}{ }_{j}\right) e_{\gamma}{ }^{k} .
$$

Эта связность единственна. Отметим, что если исходная связность $\Gamma_{\alpha \beta}{ }^{\gamma}$ симметрична, то индуцированная связность $\widehat{\Gamma}_{i j}^{k}$ также симметрична. Из уравнения (13) следует, что тензор кручения $T_{\alpha \beta}{ }^{\gamma}=\Gamma_{\alpha \beta}{ }^{\gamma}-\Gamma_{\beta \alpha}{ }^{\gamma}$ на $\mathbb{M}$ индуцирует кручение на гиперповерхности

$$
T_{i j}{ }^{k}=T_{\alpha \beta}{ }^{\gamma} e^{\alpha}{ }_{i} e^{\beta}{ }_{j} e_{\gamma}{ }^{k} .
$$

Кроме того, связность на $\mathbb{U}$ определяется единственным образом только в том случае, если на $\mathbb{M}$ помимо связности задана также метрика.

В дальнейшем все геометрические объекты, относящиеся к гиперповерхности и построенные только по индуцированной метрике $g_{i j}$ и связности $\widehat{\Gamma}_{i j}{ }^{k}$, мы будем отмечать шляпкой.

Таким образом, метрика $g_{\alpha \beta}$ и связность $\Gamma_{\alpha \beta}^{\gamma}$ на $\mathbb{M}$ индуцируют единственные метрику $g_{i j}$ и связность $\widehat{\Gamma}_{i j}{ }^{k}$ на гиперповерхности $\mathbb{U} \subset \mathbb{M}$. Обратное утверждение неверно. Если метрика и связность заданы на гиперповерхности $\mathbb{U}$, то они не индуцируют на $\mathbb{M}$ геометрию единственным образом. Это понятно, поскольку размерность гиперповерхности меньше размерности самого многообразия.

Прямые вычисления дают следующее выражение для ковариантной производной от индуцированной метрики на гиперповерхности:

$$
\widehat{\nabla}_{i} g_{j k}=\partial_{i} g_{i j}-\widehat{\Gamma}_{i j}^{l} g_{l k}-\widehat{\Gamma}_{i k}^{l} g_{j l}=\left(\nabla_{\alpha} g_{\beta \gamma}\right) e^{\alpha}{ }_{i} e^{\beta}{ }_{j} e_{k}^{\gamma} .
$$

Отсюда следует выражение для тензора неметричности на гиперповерхности:

$$
Q_{i j k}=Q_{\alpha \beta \gamma} e^{\alpha}{ }_{i} e^{\beta}{ }_{j} e^{\gamma}{ }_{k} .
$$

В частности, если связность $\Gamma_{\alpha \beta}^{\gamma}$ на $\mathbb{M}$ является метрической $\left(Q_{\alpha \beta \gamma}=0\right)$, то и индуцированная связность $\widehat{\Gamma}_{i j}{ }^{k}$ на $\mathbb{U}$ также метрическая $\left(Q_{i j k}=0\right)$.

Наличие метрики $g_{\alpha \beta}$ позволяет построить единичное векторное поле $n=n^{\alpha} \partial_{\alpha}$, ортогональное к гиперповерхности. Как уже отмечалось, система уравнений $n_{\alpha} e^{\alpha}{ }_{i}=0$ определяет 1-форму $d x^{\alpha} n_{\alpha}$ с точностью до умножения на произвольную скалярную функцию. Воспользуемся этим произволом для того, чтобы в каждой точке вектор $n^{\alpha}=g^{\alpha \beta} n_{\beta}$ имел единичную длину $(n, n)=n^{\alpha} n^{\beta} g_{\alpha \beta}=1$. По построению этот вектор ортогонален всем векторам, касательным к гиперповерхности:

$$
\left(n, e_{i}\right)=n^{\alpha} e^{\beta}{ }_{i} g_{\alpha \beta}=n_{\alpha} e^{\alpha}{ }_{i}=0 .
$$

Если на многообразии задана гиперповерхность, то естественно рассматривать базис $\left\{n, e_{i}\right\}$ в касательном пространстве $\mathbb{T}(\mathbb{M})$, определяемый этой гиперповерхностью. Этот базис определен только в точках гиперповерхности, а не на всем многообразии. Ему соответствует сопряженный базис $\left\{n=d x^{\alpha} n_{\alpha}, e^{i}=d x^{\alpha} e_{\alpha}{ }^{i}\right\}$ в кокасательном пространстве $\mathbb{T}^{*}(\mathbb{M})$. Тогда произвольный вектор $X$ и 1-форма $A$ разлагаются по этому базису:

$$
\begin{aligned}
& X^{\alpha}=X^{\perp} n^{\alpha}+X^{i} e^{\alpha}{ }_{i}, \quad X^{\perp}=X^{\alpha} n_{\alpha}, \quad X^{i}=X^{\alpha} e_{\alpha}{ }^{i}, \\
& A_{\alpha}=A_{\perp} n_{\alpha}+A_{i} e_{\alpha}{ }^{i}, \quad A_{\perp}=A_{\alpha} n^{\alpha}, \quad A_{i}=A_{\alpha} e^{\alpha}{ }_{i} \text {. }
\end{aligned}
$$


Аналогично можно разложить тензор произвольного ранга. В частности, разложение ковариантного тензора второго ранга имеет вид

$$
A_{\alpha \beta}=A_{\perp \perp} n_{\alpha} n_{\beta}+A_{\perp i} n_{\alpha} e_{\beta}^{i}+A_{i \perp} e_{\alpha}^{i} n_{\beta}+A_{i j} e_{\alpha}^{i} e_{\beta}^{j},
$$

где

$$
A_{\perp \perp}=A_{\alpha \beta} n^{\alpha} n^{\beta}, \quad A_{\perp i}=A_{\alpha \beta} n^{\alpha} e^{\beta}{ }_{i}, \quad A_{i \perp}=A_{\alpha \beta} e^{\alpha}{ }_{i} n^{\beta}, \quad A_{i j}=A_{\alpha \beta} e^{\alpha}{ }_{i} e^{\beta}{ }_{j} .
$$

Нетрудно проверить, что разложение для метрики существенно проще:

$$
g_{\alpha \beta}=n_{\alpha} n_{\beta}+e_{\alpha}^{i} e_{\beta}^{j} g_{i j} .
$$

Разложение для обратной метрики имеет аналогичный вид:

$$
g^{\alpha \beta}=n^{\alpha} n^{\beta}+e^{\alpha}{ }_{i} e^{\beta}{ }_{j} g^{i j} .
$$

Из определения обратной метрики $g^{\alpha \beta} g_{\beta \gamma}=\delta_{\gamma}^{\alpha}$ следует правило суммирования матрицы Якоби по латинским индексам:

$$
e^{\alpha}{ }_{i} e_{\beta}{ }^{i}=\delta_{\beta}^{\alpha}-n^{\alpha} n_{\beta}
$$

Из уравнения (15) вытекает, что из определения обратной индуцированной метрики $g^{i j} g_{j k}=\delta_{k}^{i}$ следует равенство

$$
e^{\alpha}{ }_{i} e_{\alpha}^{j}=\delta_{i}^{j}
$$

где суммирование проводится по греческим индексам. С учетом этого правила из (17) следует представление для обратной индуцированной метрики

$$
g^{i j}=g^{\alpha \beta} e_{\alpha}{ }^{i} e_{\beta}{ }^{j} .
$$

Метрика (16) и обратная ей (17) в базисе $n, e_{i}$ имеют блочно-диагональный вид

$$
\left(\begin{array}{cc}
1 & 0 \\
0 & g_{i j}
\end{array}\right), \quad\left(\begin{array}{cc}
1 & 0 \\
0 & g^{i j}
\end{array}\right) .
$$

С их помощью можно поднимать и опускать соответствующие индексы. Так. если $X_{\alpha}=X^{\beta} g_{\beta \alpha}$, то $X_{\perp}=X^{\perp}$ и $X_{i}=X^{j} g_{j i}$.

Индуцированная метрика (11) и связность (13) задают внутреннюю геометрию гиперповерхности $\mathbb{U} \subset \mathbb{M}$. В частности, индуцированная связность задает тензор внутренней кривизны гиперповерхности

$$
\widehat{R}_{i j k}^{l}(\widehat{\Gamma})=\partial_{i} \widehat{\Gamma}_{j k}^{l}-\widehat{\Gamma}_{i k}^{m} \widehat{\Gamma}_{j m}^{l}-(i \leftrightarrow j) .
$$

Вложение гиперповерхности $f$ позволяет определить еще один важный объект, называемый внешней кривизной гиперповерхности:

$$
K_{i j}=-\nabla_{\alpha} n_{\beta} e^{\alpha}{ }_{i} e^{\beta}{ }_{j} .
$$


Внешняя кривизна с точностью до знака равна ковариантной производной нормали, спроектированной на касательное пространство к гиперповерхности. В отличие от тензора внутренней кривизны внешняя кривизна является тензором второго ранга и в общем случае никакой симметрии по индексам не имеет. Этот тензор характеризует изменение нормали при ее параллельном переносе вдоль кривой на гиперповерхности. Раскрывая это определение, с учетом (10) получим

$$
K_{i j}=n_{\alpha}\left(\partial_{i j}^{2} x^{\alpha}+\Gamma_{\beta \gamma}{ }^{\alpha} e^{\beta}{ }_{i} e^{\gamma}{ }_{j}\right) .
$$

Антисимметричная часть тензора внешней кривизны определяется тензором кручения:

$$
K_{i j}-K_{j i}=2 K_{[i j]}=n_{\alpha} T_{\beta \gamma}{ }^{\alpha} e^{\beta}{ }_{i} e^{\gamma}{ }_{j}=T_{i j}{ }^{\perp} .
$$

Отсюда следует, что внешняя кривизна симметрична тогда и только тогда, когда связность $\Gamma_{\beta \gamma}{ }^{\alpha}$ не имеет кручения.

Вычислим ковариантную производную от матрицы Якоби

$$
\nabla_{i} e^{\alpha}{ }_{j}=e^{\beta}{ }_{i}\left(\partial_{\beta} e^{\alpha}{ }_{j}+\Gamma_{\beta \gamma}{ }^{\alpha} e^{\gamma}{ }_{j}\right)-\widehat{\Gamma}_{i j}{ }^{k} e^{\alpha}{ }_{k},
$$

где учтена связность как на всем многообразии $\Gamma_{\alpha \beta} \gamma$, так и индуцированная связность на гиперповерхности $\widehat{\Gamma}_{i j} k$.

Простые выкладки показывают, что эта ковариантная производная имеет только нормальную составляющую и пропорциональна внешней кривизне:

$$
\nabla_{i} e^{\alpha}{ }_{j}=n^{\alpha} K_{i j}
$$

Это соотношение известно как формула Гаусса-Вейнгартена. Из полученного равенства следует еще одно представление для тензора внешней кривизны:

$$
K_{i j}=n_{\alpha} \nabla_{i} e^{\alpha}{ }_{j}
$$

Полный тензор кривизны $R_{\alpha \beta \gamma \delta}$ многообразия $\mathbb{M}$, спроектированный на гиперповерхность, можно выразить через тензор внутренней кривизны $\widehat{R}_{i j k l}$, построенный только по индуцированной метрике (11) и связности (13), и тензор внешней кривизны. Для этого рассмотрим коммутатор ковариантных производных ковекторного поля, который определяется тензором кривизны и кручения:

$$
\begin{aligned}
{\left[\nabla_{\alpha}, \nabla_{\beta}\right] X_{\gamma} } & =-R_{\alpha \beta \gamma}{ }^{\delta} X_{\delta}-T_{\alpha \beta}{ }^{\delta} \nabla_{\delta} X_{\delta}= \\
& =-R_{\alpha \beta \gamma l} X^{l}-R_{\alpha \beta \gamma \perp} X^{\perp}-T_{\alpha \beta}{ }^{l} \nabla_{l} X_{\gamma}-T_{\alpha \beta}{ }^{\perp} \nabla_{\perp} X_{\gamma}
\end{aligned}
$$

где в правой части сначала вычисляются ковариантные производные, а затем проектируются на гиперповерхность и ортогональное направление: $\nabla_{l} X_{\gamma}=e^{\alpha}{ }_{l} \nabla_{\alpha} X_{\gamma}$, $\nabla_{\perp} X_{\gamma}=n^{\alpha} \nabla_{\alpha} X_{\gamma}$. Для того чтобы спроектировать это соотношение на гиперповерхность, спроектируем сначала ковариантную производную:

$$
\nabla_{i} X_{j}=e^{\alpha}{ }_{i}\left(\nabla_{\alpha} X_{\beta}\right) e^{\beta}{ }_{j}=\widehat{\nabla}_{i} X_{j}-X_{\perp} K_{i j},
$$

где

$$
\widehat{\nabla}_{i} X_{j}=\partial_{i} X_{j}-\widehat{\Gamma}_{i j}^{k} X_{k}
$$


есть $(n-1)$-мерная ковариантная производная на гиперповерхности. Аналогично проектируется вторая ковариантная производная:

$$
\begin{aligned}
\nabla_{i} \nabla_{j} X_{k} & =\widehat{\nabla}_{i} \nabla_{j} X_{k}-\nabla_{\perp} X_{k} K_{i j}-\nabla_{j} X_{\perp} K_{i k}= \\
& =\widehat{\nabla}_{i} \widehat{\nabla}_{j} X_{k}-\widehat{\nabla}_{i} X_{\perp} K_{j k}-\widehat{\nabla}_{j} X_{\perp} K_{i k}-X_{\perp} \widehat{\nabla}_{i} K_{j k}-\nabla_{\perp} X_{k} K_{i j},
\end{aligned}
$$

где

$$
\nabla_{i} K_{\perp}=e^{\alpha}{ }_{i}\left(\nabla_{\alpha} X_{\beta}\right) n^{\beta}=\widehat{\nabla}_{i} X_{\beta}+X^{j} K_{j i} .
$$

Антисимметризация полученного выражения по индексам $i, j$ дает проекцию коммутатора (25) на гиперповерхность:

$$
\left[\nabla_{i}, \nabla_{j}\right] X_{k}=-R_{i j k l} X^{l}-R_{i j k \perp} X^{\perp}-T_{i j}{ }^{l} \nabla_{l} X_{k}-T_{i j}{ }^{\perp} \nabla_{\perp} X_{k} .
$$

Учитывая независимость компонент $X^{l}$ и $X^{\perp}$ и выражения для компонент тензора кручения (14), (21), получаем выражение для проекций полного тензора кривизны на гиперповерхность:

$$
\begin{aligned}
R_{i j k l} & =\widehat{R}_{i j k l}+K_{i k} K_{j l}-K_{j k} K_{i l}, \\
R_{i j k \perp} & =\widehat{\nabla}_{i} K_{j k}-\widehat{\nabla}_{j} K_{i k}+T_{i j}{ }^{l} K_{l k} .
\end{aligned}
$$

Полученные соотношения являются обобщением уравнений Гаусса-Петерсона-Кодацци на случай, когда на объемлющем многообразии $\mathbb{M}$ задана не риманова геометрия, а произвольная аффинная геометрия с ненулевым кручением и неметричностью.

В заключение настоящего раздела вычислим нормальные компоненты $G_{\perp \perp}$ и $G_{\perp i}$ тензора Эйнштейна

$$
G_{\alpha \beta}=R_{\alpha \beta}-\frac{1}{2} g_{\alpha \beta} R
$$

в римановой геометрии, когда кручение и неметричность равны нулю. Сначала вычислим скалярную кривизну

$$
R=g^{\alpha \gamma} g^{\beta \delta} R_{\alpha \beta \gamma \delta}=2 R_{\perp \perp}+g^{i k} g^{j l} R_{i j k l},
$$

где $R_{\perp \perp}=g^{i j} R_{i \perp j}-$ нормальная составляющая тензора Риччи и учтено представление для обратной метрики (17). С учетом уравнений Гаусса-Петерсона-Кодацци (26) получаем

$$
g^{i k} g^{j l} R_{i j k l}=\widehat{R}+K^{2}-K^{i j} K_{i j},
$$

где $\widehat{R}$ - скалярная внутренняя кривизна гиперповерхности, $K=g^{i j} K_{i j}$ - скалярная внешняя кривизна гиперповерхности. Отсюда следуют выражения для нормальных компонент тензора Эйнштейна:

$$
\begin{aligned}
G_{\perp \perp} & =-\frac{1}{2}\left(\widehat{R}+K^{2}-K^{i j} K_{i j}\right), \\
G_{\perp i} & =\widehat{\nabla}_{j} K_{i}{ }^{j}-\nabla_{i} K .
\end{aligned}
$$

Важным обстоятельством является то, что эти компоненты тензора Эйнштейна вообще не содержат нормальных производных к гиперповерхности $\nabla_{\perp}$ от индуцированной метрики и тензора внешней кривизны. На гамильтоновом языке это означает отсутствие производных по времени и то, что уравнения Эйнштейна $G_{\perp \perp}=0$ 
и $G_{\perp i}=0$ представляют собой связи, поскольку компоненты тензора внешней кривизны $K^{i j}$, как будет показано в следующем разделе, пропорциональны импульсам, канонически сопряженным компонентам индуцированной метрики $g_{i j}$.

\section{4. КРИВИЗНА В АДМ-ПАРАМЕТРИЗАЦИИ МЕТРИКИ}

АДМ-параметризация метрики (1) удобна для канонической формулировки ОТО, в которой независимыми переменными являются компоненты метрики $g_{\alpha \beta}$ и канонически сопряженные импульсы $p^{\alpha \beta}$. Однако чтобы перейти от лагранжиана к гамильтониану, необходимо произвести довольно громоздкие вычисления, чем мы и займемся в настоящем разделе.

Для существенного упрощения вычислений следует воспользоваться результатами предыдущего раздела. А именно, сечения $x^{0}=$ const пространства-времени $\mathbb{M}$ задают семейства гиперповерхностей $\mathbb{U} \subset \mathbb{M}$, которые по предположению являются пространственноподобными. В качестве координат на гиперповерхностях выберем пространственные координаты

$$
\left\{u^{i}\right\} \rightarrow\left\{x^{\mu}\right\}
$$

При этом мы теряем возможность независимого преобразования координат в пространстве-времени $\mathbb{M}$ и на пространственноподобной гиперповерхности $\mathbb{U}$, зато многие формулы упрощаются.

Матрица Якоби вложения гиперповерхности в рассматриваемом случае имеет вид

$$
\left\{e^{\alpha}{ }_{i}\right\} \rightarrow\left\{0_{\nu}, \delta_{\nu}^{\mu}\right\}, \quad\left\{e_{\alpha}{ }^{i}\right\} \rightarrow\left\{N^{\mu}, \delta_{\nu}^{\mu}\right\}
$$

где $0_{\nu}$ обозначает строку, состоящую из $n-1$ нулей. Вложение индуцирует на гиперповерхностях метрику $g_{\mu \nu}$ согласно формуле (11).

Построим векторное поле $n=n^{\alpha} \partial_{\alpha}$, ортогональное семейству пространственных гиперповерхностей $x^{0}=$ const. Из условия ортогональности $(n, X)=0$, где $X=$ $X^{\mu} \partial_{\mu}$ - произвольный вектор, касательный к сечению $x^{0}=0$, следует, что

$$
n=n^{0}\left(\partial_{0}-N^{\mu} \partial_{\mu}\right) .
$$

Если, кроме того, положить $n^{0}=1 / N$, то длина нормального вектора будет равна единице $\left(n^{2}=1\right)$. Таким образом, единичный вектор, нормальный к сечению $x^{0}=$ const, имеет вид

$$
n=\frac{1}{N}\left(\partial_{0}-N^{\mu} \partial_{\mu}\right)
$$

и всегда является времениподобным. Соответствующая ортонормальная 1-форма имеет вид

$$
n=d x^{0} N
$$

Произвольный тензор на $\mathbb{M}$ можно разложить по базису $\left\{n, e_{\mu}\right\}$. В частности, для векторов и 1-форм справедливы разложения

$$
X^{\alpha}=X^{\perp} n^{\alpha}+\tilde{X}^{\mu} e_{\mu}{ }^{\alpha}, \quad X_{\alpha}=X_{\perp} n_{\alpha}+\tilde{X}_{\mu} e^{\mu}{ }_{\alpha},
$$


где

$$
\begin{aligned}
X^{\perp} & =X^{0} N, & \widetilde{X}^{\mu} & =X^{0} N^{\mu}+X^{\mu}, \\
X_{\perp} & =\frac{1}{N}\left(X_{0}-N^{\mu} X_{\mu}\right), & \widetilde{X}_{\mu} & =X_{\mu} .
\end{aligned}
$$

Представления для метрики (16) всего пространства-времени и ее обратной (17) принимают вид

$$
\begin{aligned}
& g_{\alpha \beta}=n_{\alpha} n_{\beta}+g_{\mu \nu} e_{\alpha}{ }^{\mu} e_{\beta}{ }^{\nu}, \\
& g^{\alpha \beta}=n^{\alpha} n^{\beta}+\hat{g}^{\mu \nu} e_{\mu}{ }^{\alpha} e_{\nu}{ }^{\beta} .
\end{aligned}
$$

Связность (13), индуцированная на гиперповерхностях - это символы Кристоффеля $\widehat{\Gamma}_{\mu \nu}{ }^{\rho}$, построенные по пространственной метрике $g_{\mu \nu}$.

Тензор внешней кривизны (20) гиперповерхности $x^{0}=$ const в АДМ-параметризации метрики имеет вид

$$
K_{\mu \nu}=\Gamma_{\mu \nu}^{0} N=\frac{1}{2 N}\left(\widehat{\nabla}_{\mu} N_{\nu}+\widehat{\nabla}_{\nu} N_{\mu}-\dot{g}_{\mu \nu}\right),
$$

где точка обозначает дифференцирование по времени, $\dot{g}_{\mu \nu}=\partial_{0} g_{\mu \nu}$, и $\widehat{\nabla}_{\mu} N_{\nu}=$ $\partial_{\mu} N_{\nu}-\widehat{\Gamma}_{\mu \nu}{ }^{\rho} N_{\rho}$. Тензор внешней кривизны симметричен $\left(K_{\mu \nu}=K_{\nu \mu}\right)$, поскольку кручение в метрической формулировке ОТО равно нулю. В дальнейшем нам понадобится также след тензора внешней кривизны

$$
K=K_{\mu}{ }^{\mu}=\hat{g}^{\mu \nu} K_{\mu \nu} .
$$

При вычислении тензора кривизны $R_{\alpha \beta \gamma \delta}$ пространства-времени $\mathbb{M}$ все производные по времени от пространственной части метрики $\dot{g}_{\mu \nu}$ удобно выражать через $K_{\mu \nu}$. Кроме этого, для исключения вторых производных по времени $\ddot{g}_{\mu \nu}$ нам понадобится производная по времени от тензора внешней кривизны

$$
\dot{K}_{\mu \nu}=\frac{1}{2 N}\left[\widehat{\nabla}_{\mu} \dot{N}_{\nu}+\widehat{\nabla}_{\nu} \dot{N}_{\mu}-\ddot{g}_{\mu \nu}-N^{\rho}\left(\widehat{\nabla}_{\mu} \dot{g}_{\nu \rho}+\widehat{\nabla}_{\nu} \dot{g}_{\mu \rho}-\widehat{\nabla}_{\rho} \dot{g}_{\mu \nu}\right)\right]-\frac{\dot{N}}{N} K_{\mu \nu}
$$

где

$$
\widehat{\nabla}_{\mu} \dot{N}_{\nu}=\partial_{\mu} \dot{N}_{\nu}-\widehat{\Gamma}_{\mu \nu}^{\rho} \dot{N}_{\rho}
$$

и куда при вычислениях мы должны подставить выражение для $\dot{g}_{\mu \nu}$ через $K_{\mu \nu}$.

Приступим к вычислению тензора кривизны $R_{\alpha \beta \gamma \delta}$ для метрики, записанной в виде (1). Прямые выкладки приводят к следующим выражениям для линейно независимых символов Кристоффеля:

$$
\begin{aligned}
\Gamma_{00}{ }^{0} & =\frac{1}{N}\left(\dot{N}+N^{\rho} \partial_{\rho} N+N^{\rho} N^{\sigma} K_{\rho \sigma}\right), \\
\Gamma_{00}{ }^{\mu} & =\hat{g}^{\mu \nu}\left(\dot{N}_{\nu}-N \partial_{\nu} N-N^{\rho} \widehat{\nabla}_{\nu} N_{\rho}\right)-\frac{N^{\mu}}{N}\left(\dot{N}+N^{\rho} \partial_{\rho} N+N^{\rho} N^{\sigma} K_{\rho \sigma}\right), \\
\Gamma_{0 \mu}{ }^{0} & =\frac{1}{N}\left(\partial_{\mu} N+N^{\nu} K_{\mu \nu}\right), \\
\Gamma_{0 \mu}{ }^{\nu} & =\widehat{\nabla}_{\mu} N^{\nu}-N K_{\mu}{ }^{\nu}-\frac{N^{\nu}}{N}\left(\partial_{\mu} N+N^{\rho} K_{\mu \rho}\right), \\
\Gamma_{\mu \nu}{ }^{0} & =\frac{1}{N} K_{\mu \nu}, \\
\Gamma_{\mu \nu}{ }^{\rho} & =\widehat{\Gamma}_{\mu \nu}{ }^{\rho}-\frac{N^{\rho}}{N} K_{\mu \nu} .
\end{aligned}
$$


В дальнейшем нам понадобятся следующие комбинации символов Кристоффеля:

$$
\Gamma_{\alpha}=\Gamma_{\alpha \beta}^{\beta}, \quad g^{\beta \gamma} \Gamma_{\beta \gamma}{ }^{\alpha} .
$$

Несложные вычисления дают

$$
\begin{aligned}
\Gamma_{0} & =\frac{\dot{N}}{N}+\widehat{\nabla}_{\mu} N^{\mu}-N K \\
\Gamma_{\mu} & =\widehat{\Gamma}_{\mu}+\frac{\partial_{\mu} N}{N}, \\
g^{\beta \gamma} \Gamma_{\beta \gamma}{ }^{0}= & \frac{1}{N} K+\frac{1}{N^{3}}\left(\dot{N}-N^{\mu} \partial_{\mu} N\right), \\
g^{\beta \gamma} \Gamma_{\beta \gamma}{ }^{\mu}= & \left(\hat{g}^{\rho \sigma}+\frac{N^{\rho} N^{\sigma}}{N^{2}}\right) \widehat{\Gamma}_{\rho \sigma}^{\mu}-\frac{N^{\mu}}{N} K-\frac{N^{\mu}}{N^{3}}\left(\dot{N}-N^{\rho} \partial_{\rho} N\right)+ \\
& \quad+\frac{1}{N^{2}} \hat{g}^{\mu \rho}\left(\dot{N}_{\rho}-N \partial_{\rho} N-N^{\sigma} \widehat{\nabla}_{\rho} N_{\sigma}-2 N^{\sigma} \widehat{\nabla}_{\sigma} N_{\rho}+2 N N^{\sigma} K_{\rho \sigma}\right) .
\end{aligned}
$$

Выпишем также формулы дифференцирования по времени для символов Кристоффеля

$$
\begin{aligned}
\partial_{0} \widehat{\Gamma}_{\mu \nu \rho} & =\frac{1}{2}\left(\widehat{\nabla}_{\mu} \dot{g}_{\nu \rho}+\widehat{\nabla}_{\nu} \dot{g}_{\mu \rho}-\widehat{\nabla}_{\rho} \dot{g}_{\mu \nu}\right)+\widehat{\Gamma}_{\mu \nu}^{\sigma} \dot{g}_{\rho \sigma}, \\
\partial_{0} \widehat{\Gamma}_{\mu \nu} \sigma & =\frac{1}{2} \hat{g}^{\sigma \rho}\left(\widehat{\nabla}_{\mu} \dot{g}_{\nu \rho}+\widehat{\nabla}_{\nu} \dot{g}_{\mu \rho}-\widehat{\nabla}_{\rho} \dot{g}_{\mu \nu}\right), \\
\partial_{0} \widehat{\Gamma}_{\mu} & =\frac{1}{2} \hat{g}^{\nu \rho} \widehat{\nabla}_{\mu} \dot{g}_{\nu \rho} .
\end{aligned}
$$

В этих выражениях производные по времени $\dot{g}_{\mu \nu}$ также исключаются с помощью соотношения (32).

Теперь вычислим линейно независимые компоненты тензора кривизны:

$$
\begin{aligned}
R_{0 \mu 0 \nu}=- & N \dot{K}_{\mu \nu}+\widehat{R}_{\mu \rho \nu \sigma} N^{\rho} N^{\sigma}+N N^{\rho}\left(\widehat{\nabla}_{\mu} K_{\nu \rho}+\widehat{\nabla}_{\nu} K_{\mu \rho}-\widehat{\nabla}_{\rho} K_{\mu \nu}\right)+ \\
& +N \widehat{\nabla}_{\mu} \widehat{\nabla}_{\nu} N+K_{\mu \nu} N^{\rho} N^{\sigma} K_{\rho \sigma}+N\left(K_{\mu}{ }^{\rho} \widehat{\nabla}_{\nu} N_{\rho}+K_{\nu}{ }^{\rho} \widehat{\nabla}_{\mu} N_{\rho}\right)- \\
& \quad-N^{2} K_{\mu}{ }^{\rho} K_{\nu \rho}-N^{\rho} N^{\sigma} K_{\mu \rho} K_{\nu \sigma}, \\
R_{\mu \nu \rho 0}= & \widehat{R}_{\mu \nu \rho \sigma} N^{\sigma}+N\left(\widehat{\nabla}_{\mu} K_{\nu \rho}-\widehat{\nabla}_{\nu} K_{\mu \rho}\right)+\left(K_{\mu \rho} K_{\nu \sigma}-K_{\nu \rho} K_{\mu \sigma}\right) N^{\sigma} \\
R_{\mu \nu \rho \sigma}= & \widehat{R}_{\mu \nu \rho \sigma}+K_{\mu \rho} K_{\nu \sigma}-K_{\mu \sigma} K_{\nu \rho},
\end{aligned}
$$

где мы воспользовались формулой для коммутатора ковариантных производных

$$
\left(\widehat{\nabla}_{\mu} \widehat{\nabla}_{\nu}-\widehat{\nabla}_{\nu} \widehat{\nabla}_{\mu}\right) N_{\rho}=-\widehat{R}_{\mu \nu \rho \sigma} N^{\sigma} .
$$

Компоненты тензора кривизны, имеющие по крайней мере один временно́й индекс, выглядят проще относительно базиса $n, e_{\mu}$ :

$$
\begin{aligned}
R_{\perp \mu \perp \nu}= & \frac{1}{N}\left(-\dot{K}_{\mu \nu}+\widehat{\nabla}_{\mu} \widehat{\nabla}_{\nu} N+K_{\mu \rho} \widehat{\nabla}_{\nu} N^{\rho}+\right. \\
& \left.\quad+K_{\nu \rho} \widehat{\nabla}_{\mu} N^{\rho}-N K_{\mu \rho} K_{\nu}{ }^{\rho}+N^{\rho} \widehat{\nabla}_{\rho} K_{\mu \nu}\right) \\
R_{\mu \nu \rho \perp}= & \widehat{\nabla}_{\mu} K_{\nu \rho}-\widehat{\nabla}_{\nu} K_{\mu \rho} .
\end{aligned}
$$


Компоненты тензора кривизны $R_{\mu \nu \rho \sigma}$ и $R_{\mu \nu \rho \perp}$ фактически уже были получены в предыдущем разделе (см. (26), (27)) без прямых вычислений.

Тензор Риччи имеет следующие линейно независимые компоненты:

$$
\begin{aligned}
R_{00}=- & N \hat{g}^{\mu \nu} \dot{K}_{\mu \nu}+\widehat{R}_{\mu \nu} N^{\mu} N^{\nu}+N N^{\mu}\left(2 \widehat{\nabla}_{\nu} K^{\nu}{ }_{\mu}-\partial_{\mu} K\right)+N \widehat{\nabla}^{\mu} \widehat{\nabla}_{\mu} N+ \\
& +N^{\mu} N^{\nu} K_{\mu \nu} K+2 N K^{\mu \nu} \widehat{\nabla}_{\mu} N_{\nu}-N^{2} K^{\mu \nu} K_{\mu \nu}-2 N^{\mu} N^{\nu} K_{\mu}{ }^{\rho} K_{\nu \rho}+ \\
& +\frac{N^{\mu} N^{\nu}}{N}\left(-\dot{K}_{\mu \nu}+N^{\rho} \widehat{\nabla}_{\mu} K_{\nu \rho}+\widehat{\nabla}_{\mu} \widehat{\nabla}_{\nu} N+2 K_{\mu}{ }^{\rho} \widehat{\nabla}_{\nu} N_{\rho}\right), \\
R_{0 \mu}= & \frac{N^{\nu}}{N}\left(-\dot{K}_{\mu \nu}+N^{\sigma} \widehat{\nabla}_{\nu} K_{\mu \sigma}+\widehat{\nabla}_{\mu} \widehat{\nabla}_{\nu} N+K_{\mu}{ }^{\rho} \widehat{\nabla}_{\nu} N_{\rho}+K_{\nu}{ }^{\rho} \widehat{\nabla}_{\mu} N_{\rho}\right)+ \\
& +\widehat{R}_{\mu \nu} N^{\nu}+N\left(\widehat{\nabla}_{\nu} K_{\mu}^{\nu}-\partial_{\mu} K\right)+K_{\mu \nu} N^{\nu} K-2 K_{\mu}{ }^{\rho} K_{\nu \rho} N^{\nu}, \\
R_{\mu \nu}= & \widehat{R}_{\mu \nu}+\frac{1}{N}\left(-\dot{K}_{\mu \nu}+\widehat{\nabla}_{\mu} \widehat{\nabla}_{\nu} N+K_{\mu}{ }^{\rho} \widehat{\nabla}_{\nu} N_{\rho}+K_{\nu}{ }^{\rho} \widehat{\nabla}_{\mu} N_{\rho}\right)+ \\
& +\frac{N^{\rho}}{N} \widehat{\nabla}_{\rho} K_{\mu \nu}+K_{\mu \nu} K-2 K_{\mu}{ }^{\rho} K_{\nu \rho} .
\end{aligned}
$$

Приведем также для справки компоненты тензора Риччи относительно базиса $n, e_{\mu}$ :

$$
\begin{aligned}
R_{\perp \perp} & =-\frac{1}{N} \hat{g}^{\mu \nu} \dot{K}_{\mu \nu}+\frac{1}{N} \widehat{\nabla}^{\mu} \widehat{\nabla}_{\mu} N+\frac{2}{N} K^{\mu \nu} \widehat{\nabla}_{\mu} N_{\nu}-K^{\mu \nu} K_{\mu \nu}+\frac{N^{\mu}}{N} \partial_{\mu} K, \\
R_{\perp \mu} & =\widehat{\nabla}_{\nu} K^{\nu}{ }_{\mu}-\partial_{\mu} K .
\end{aligned}
$$

Наконец, вычисляем скалярную кривизну:

$$
R=\widehat{R}+\frac{2}{N}\left(-\hat{g}^{\mu \nu} \dot{K}_{\mu \nu}+\widehat{\nabla}^{\mu} \widehat{\nabla}_{\mu} N+2 K^{\mu \nu} \widehat{\nabla}_{\mu} N_{\nu}+N^{\mu} \partial_{\mu} K\right)-3 K^{\mu \nu} K_{\mu \nu}+K^{2} .
$$

\section{5. ГАМИЛЬТОНИАН}

Скалярная кривизна содержит вторые производные от компонент метрики как по времени, так и по пространственным координатам, и поэтому неудобна для канонической формулировки ОТО. Для канонической формулировки достаточно исключить из лагранжиана только вторые производные по времени. Наиболее простой вид лагранжева плотность принимает после добавления следующего граничного члена:

$$
\mathcal{L}_{\mathrm{ADM}}=N \hat{e} R+2 \partial_{0}(\hat{e} K)-2 \partial_{\mu}\left(\hat{e} \hat{g}^{\mu \nu} \partial_{\nu} N\right) .
$$

Прямые вычисления приводят к выражению

$$
\mathcal{L}_{\mathrm{ADM}}=N \hat{e}\left(K^{\mu \nu} K_{\mu \nu}-K^{2}+\widehat{R}\right) .
$$

Теперь нетрудно перейти к гамильтонову формализму. Во-первых, АДМ-лагранжиан не содержит производных по времени от функции хода $N$ и функций сдвига $N_{\mu}$. Это значит, что теория содержит $n$ первичных связей

$$
p^{\perp}=\frac{\partial \mathcal{L}_{\mathrm{ADM}}}{\partial \dot{N}}=0, \quad p^{\mu}=\frac{\partial \mathcal{L}_{\mathrm{ADM}}}{\partial \dot{N}_{\mu}}=0,
$$

число которых совпадает с числом независимых функций, параметризующих диффеоморфизмы. 
Импульсы, канонически сопряженные к пространственной метрике $g_{\mu \nu}$, пропорциональны тензору внешней кривизны:

$$
p^{\mu \nu}=\frac{\partial \mathcal{L}_{\mathrm{ADM}}}{\partial \dot{g}_{\mu \nu}}=-\frac{1}{2 N} \frac{\partial \mathcal{L}_{\mathrm{ADM}}}{\partial \dot{K}_{\mu \nu}}=-\hat{e}\left(K^{\mu \nu}-\hat{g}^{\mu \nu} K\right) .
$$

Отметим, что импульсы являются не тензорами относительно преобразований координат $x^{\mu}$, а тензорными плотностями степени -1 , как и определитель репера, степень которого по определению равна

$$
\operatorname{deg} \hat{e}=-1
$$

Чтобы исключить из АДМ-лагранжиана скорости $\dot{g}_{\mu \nu}$, разложим импульсы на неприводимые компоненты, выделив из $p^{\mu \nu}$ след:

$$
p^{\mu \nu}=\tilde{p}^{\mu \nu}+\frac{1}{n-1} p \hat{g}^{\mu \nu},
$$

где мы ввели след импульсов

$$
p=p^{\mu \nu} g_{\mu \nu}=\hat{e}(n-2) K
$$

и симметричную бесследовую часть

$$
\tilde{p}^{\mu \nu}=\tilde{p}^{\nu \mu}=-\hat{e}\left(K^{\mu \nu}-\frac{1}{n-1} \hat{g}^{\mu \nu} K\right), \quad \tilde{p}^{\mu \nu} g_{\mu \nu}=0 .
$$

Теперь можно решить уравнение (44) относительно скоростей, воспользовавшись соотношением (32):

$$
\dot{g}_{\mu \nu}=\frac{2 N}{\hat{e}}\left(p_{\mu \nu}-\frac{1}{(n-2)} p g_{\mu \nu}\right)+\widehat{\nabla}_{\mu} N_{\nu}+\widehat{\nabla}_{\nu} N_{\mu} .
$$

Несложные вычисления приводят к гамильтоновой плотности

$$
\mathcal{H}=p^{\mu \nu} \dot{g}_{\mu \nu}-\mathcal{L}_{\mathrm{ADM}}=N H_{\perp}+N^{\mu} H_{\mu}+2 \partial_{\mu}\left(p^{\mu \nu} N_{\nu}\right),
$$

где

$$
\begin{aligned}
& H_{\perp}=\frac{1}{\hat{e}}\left(p^{\mu \nu} p_{\mu \nu}-\frac{1}{(n-2)} p^{2}\right)-\hat{e} \widehat{R}, \\
& H_{\mu}=-2 \widehat{\nabla}_{\nu} p^{\nu}{ }_{\mu}=-2 \partial_{\nu} p_{\mu}^{\nu}+\partial_{\mu} g_{\nu \rho} p^{\nu \rho}
\end{aligned}
$$

и $p_{\mu \nu}=g_{\mu \rho} g_{\nu \sigma} p^{\rho \sigma}$. Отметим, что ковариантная производная от импульсов содержит только одно слагаемое с символами Кристоффеля, так как импульсы являются тензорными плотностями. Выражения для $H_{\perp}$ и $H_{\mu}$ пропорциональны соответственно компонентам $G_{\perp \perp}$ и $G_{\perp \mu}$ тензора Эйнштейна $(28)$, и этим оправдываются выбранные обозначения.

Отбрасывая в выражении для гамильтоновой плотности (47) дивергенцию, приходим к окончательному выражению для гамильтониана

$$
H_{\mathrm{ADM}}=\int d \mathbf{x} \mathcal{H}_{\mathrm{ADM}}=\int d \mathbf{x}\left(N H_{\perp}+N^{\mu} H_{\mu}\right)
$$


Перепишем выражение для $H_{\perp}$ в терминах неприводимых компонент импульсов:

$$
H_{\perp}=\frac{1}{\hat{e}}\left[\tilde{p}^{\mu \nu} \tilde{p}_{\mu \nu}-\frac{1}{(n-1)(n-2)} p^{2}\right]-\hat{e} \widehat{R} .
$$

Отсюда, в частности, следует, что квадратичная форма импульсов в $H_{\perp}$ при $n \geqslant 3$ не является положительно определенной.

\section{6. ВТОРИЧНЫЕ СВЯЗИ}

Для завершения построения гамильтонова формализма необходимо исследовать согласованность первичных связей (43) с уравнениями движения. Фазовое пространство ОТО описывается $n(n+1)$ сопряженными координатами и импульсами: $\left(N, p^{\perp}\right), \quad\left(N_{\mu}, p^{\mu}\right), \quad\left(g_{\mu \nu}, p^{\mu \nu}\right) ;$ на нем задана каноническая одновременна́я скобка Пуассона

$$
\left[N, p^{\perp}\right]=\delta, \quad\left[N_{\mu}, p^{\prime \nu}\right]=\delta_{\mu}^{\nu} \delta, \quad\left[g_{\mu \nu}, p^{\rho \sigma}\right]=\delta_{\mu \nu}^{\rho \sigma} \delta,
$$

где штрих у полевой переменной означает, что она рассматривается в точке $\mathbf{x}^{\prime}=$ $\left(x^{\prime 1}, \ldots, x^{\prime n-1}\right)$. Все поля рассматриваются в один момент времени $t=x^{0}$. В правых частях скобок Пуассона для краткости использованы следующие обозначения для $(n-1)$-мерной $\delta$-функции и симметризованной комбинации символов Кронекера:

$$
\begin{aligned}
\delta=\delta^{(n-1)}\left(\mathbf{x}-\mathbf{x}^{\prime}\right) & =\delta\left(x^{1}-x^{\prime 1}\right) \ldots \delta\left(x^{n-1}-x^{\prime n-1}\right) \\
\delta_{\mu \nu}^{\rho \sigma} & =\frac{1}{2}\left(\delta_{\mu}^{\rho} \delta_{\nu}^{\sigma}+\delta_{\mu}^{\sigma} \delta_{\nu}^{\rho}\right) .
\end{aligned}
$$

Чтобы отличать $\delta$-функцию от вариации поля, мы будем писать все $\delta$-функции справа.

Рассмотрим теперь гамильтоновы уравнения движения для первичных связей (43):

$$
\dot{p}^{\perp}=\left[p^{\perp}, H_{\mathrm{ADM}}\right]=-H_{\perp}, \quad \dot{p}^{\mu}=\left[p^{\mu}, H_{\mathrm{ADM}}\right]=-H^{\mu} .
$$

Из условия согласованности первичных связей с уравнениями движения $\dot{p}^{\perp}=0$, $\dot{p}^{\mu}=0$ следуют вторичные связи

$$
H_{\perp}=0, \quad H_{\mu}=0,
$$

где $H_{\perp}=H^{\perp}$ и $H_{\mu}=g_{\mu \nu} H^{\nu}$. Отметим, что вторичные связи являются не тензорами, а тензорными плотностями степени -1. Кроме того, вместо связей $H^{\mu}$ удобнее рассматривать эквивалентную систему связей с опущенным индексом $H_{\mu}$. Ниже будет показано, что эти связи определяют генераторы преобразований координат на сечениях $x^{0}=$ const и удовлетворяют более простой алгебре.

Связи $H_{\mu}$ линейны по импульсам и метрике. Связь $H_{\perp}$ квадратична по импульсам и неполиномиальна по метрике $g_{\mu \nu}$, поскольку зависит от корня из определителя метрики $\hat{e}$ и обратной метрики $\hat{g}^{\mu \nu}$. Последнее обстоятельство является существенной технической трудностью при построении теории возмущений.

Вторичные связи не зависят от канонических переменных $\left(N, p^{\perp}\right),\left(N_{\mu}, p^{\mu}\right)$, и их можно исключить, рассматривая $n(n-1)$-мерное фазовое пространство переменных 
$g_{\mu \nu}$ и $p^{\mu \nu}$, на которые наложены связи (52). В этом случае функция хода $N$ и функции сдвига $N_{\mu}$ рассматриваются как множители Лагранжа в задаче на условный экстремум для действия

$$
S=\int d^{n} x\left(p^{\mu \nu} \dot{g}_{\mu \nu}-\mathcal{H}_{\mathrm{ADM}}\right)
$$

Поскольку гамильтониан ОТО (49) представляет собой линейную комбинацию связей, то для исследования согласованности вторичных связей (52) с уравнениями движения необходимо вычислить скобки Пуассона связей между собой. Алгебра связей в ОТО хорошо известна:

$$
\begin{aligned}
{\left[H_{\perp}, H_{\perp}^{\prime}\right] } & =-\left(H_{\mu} \hat{g}^{\mu \nu}+H_{\mu}^{\prime} \hat{g}^{\prime \mu \nu}\right) \delta_{\nu}, \\
{\left[H_{\perp}, H_{\mu}^{\prime}\right] } & =-H_{\perp}^{\prime} \delta_{\mu} \\
{\left[H_{\mu}, H_{\nu}^{\prime}\right] } & =-H_{\nu} \delta_{\mu}-H_{\mu}^{\prime} \delta_{\nu},
\end{aligned}
$$

где введено сокращенное обозначение для производной $\delta$-функции

$$
\delta_{\mu}=\frac{\partial}{\partial x^{\prime \mu}} \delta\left(x^{\prime}-x\right)
$$

Прямое вычисление алгебры связей (53)-(55) очень громоздко. Эта алгебра впервые была выписана Дираком [14] из соображений симметрии. При этом были сделаны предположения о виде связей, которые не выполняются в ОТО, и вопрос о существовании соответствующего канонического преобразования в настоящее время открыт. Поэтому получение алгебры связей Дираком нельзя считать удовлетворительным.

Вид двух скобок Пуассона (54) и (55) действительно можно найти, не прибегая к прямым вычислениям. С этой целью рассмотрим функционал

$$
T_{u}=-\int d \mathbf{x} u^{\mu} H_{\mu}
$$

где $u^{\mu}(x)$ - малое векторное поле. Вычисление скобок Пуассона координат фазового пространства $g_{\mu \nu}, p^{\mu \nu}$ с $T_{u}$ дает

$$
\begin{aligned}
& \delta_{u} g_{\mu \nu}=\left[g_{\mu \nu}, T_{u}\right]=-\partial_{\mu} u^{\rho} g_{\rho \nu}-\partial_{\nu} u^{\rho} g_{\mu \rho}-u^{\rho} \partial_{\rho} g_{\mu \nu}, \\
& \delta_{u} p^{\mu \nu}=\left[p^{\mu \nu}, T_{u}\right]=\partial_{\rho} u^{\mu} p^{\rho \nu}+\partial_{\rho} u^{\nu} p^{\mu \rho}-\partial_{\rho}\left(u^{\rho} p^{\mu \nu}\right) .
\end{aligned}
$$

Это значит, что функционал $T_{u}$, который определяется связями $H_{\mu}$, является генератором общих преобразований координат на гиперповерхностях $x^{0}=$ const. Haпомним, что импульсы $p^{\mu \nu}$ являются тензорными плотностями степени -1 . Алгебра преобразований координат хорошо известна и задается скобкой Пуассона (55). Скобку Пуассона (54) также можно не вычислять явно. Ее вид следует из того, что связь $H_{\perp}$ является скалярной плотностью степени -1 . Таким образом, необходимо вычислить только скобку Пуассона (53). Эти вычисления очень громоздки и впервые, по-видимому, были проделаны де Виттом [4]. В следующем разделе мы 
вычислим эту скобку после канонического преобразования, приводящего связи к полиномиальному виду, что существенно упрощает вычисления.

Ввиду того, что связи $H_{\mu}$ задают только пространственные диффеоморфизмы, мы будем называть их кинематическими. Они также не зависят от констант связи в действии, если таковые имеются. Связь $H_{\perp}$ называется динамической, так как она определяет развитие начальных данных во времени и существенно зависит от исходного действия, в частности от констант связи.

Для сравнения приведем скобку Пуассона связей $H^{\mu}=0$ с контравариантным индексом, которые эквивалентны связям $H_{\mu}=0$ :

$$
\left[H^{\mu}, H^{\prime \nu}\right]=\left(\hat{g}^{\mu \nu} H^{\rho}+\hat{g}^{\prime \mu \nu} H^{\prime \rho}\right) \delta_{\rho}+\left(\hat{g}^{\mu \rho} \partial_{\rho} g_{\sigma \lambda} \hat{g}^{\nu \sigma}-\hat{g}^{\nu \rho} \partial_{\rho} g_{\sigma \lambda} \hat{g}^{\mu \sigma}\right) H^{\lambda} \delta
$$

Как видим, эта скобка Пуассона выглядит сложнее скобки (55).

\section{7. КАНОНИЧЕСКОЕ ПРЕОБРАЗОВАНИЕ}

Идея канонического преобразования состоит в следующем. Импульсы $p^{\mu \nu}$ приводимы и разлагаются на бесследовую часть и след (45). При вычислениях, как правило, удобнее работать с неприводимыми компонентами, поскольку много слагаемых автоматически сокращается. Поставим вопрос: нельзя ли совершить такое каноническое преобразование, после которого новыми импульсами будут неприводимые компоненты $\tilde{p}^{\mu \nu}$ и $p$ ? Этот вопрос нетривиален, потому что разложение импульсов включает метрику, компоненты которой сами являются координатами фазового пространства. Ответ на поставленный вопрос отрицателен, потому что скобка Пуассона импульсов между собой отлична от нуля. Например, $\left[\tilde{p}^{\mu \nu}, p^{\prime}\right] \neq 0$. Однако существует такое каноническое преобразование, что новые импульсы будут пропорциональны неприводимым компонентам $\tilde{p}^{\mu \nu}$ и $p$. Построением этого канонического преобразования мы и займемся в настоящем разделе.

Рассмотрим каноническое преобразование

$$
\left(g_{\mu \nu}, p^{\mu \nu}\right) \rightarrow\left(k_{\mu \nu}, P^{\mu \nu}\right),(\rho, P)
$$

к новым парам канонически сопряженных координат и импульсов, где на координаты $k_{\mu \nu}=k_{\nu \mu}$ и сопряженные импульсы $P^{\mu \nu}=P^{\nu \mu}$ наложены дополнительные условия

$$
\left|\operatorname{det} k_{\mu \nu}\right|=1, \quad P^{\mu \nu} k_{\mu \nu}=0
$$

и $\rho>0$. В качестве производящего функционала канонического преобразования выберем интеграл по пространству

$$
F=-\int d \mathbf{x} \rho^{m} k_{\mu \nu} p^{\mu \nu}, \quad m \in \mathbb{R}, \quad m \neq 0,
$$

зависящий от новых координат $\rho, k_{\mu \nu}$ и старых импульсов $p^{\mu \nu}$, а также от вещественного параметра $m$. Тогда старые координаты и новые импульсы определяются 
вариационными производными (см., например, [15])

$$
\begin{aligned}
g_{\mu \nu} & =-\frac{\delta F}{\delta p^{\mu \nu}}=\rho^{m} k_{\mu \nu}, \\
P^{\mu \nu} & =-\frac{\delta F}{\delta k_{\mu \nu}}=\rho^{m} \tilde{p}^{\mu \nu}, \\
P & =-\frac{\delta F}{\delta \rho}=\frac{m}{\rho} p .
\end{aligned}
$$

При вычислении вариационной производной по $k_{\mu \nu}$ учтено условие $\left|\operatorname{det} k_{\mu \nu}\right|=1$, из которого вытекает ограничение на вариации $k^{\mu \nu} \delta k_{\mu \nu}=0$, где $k^{\mu \nu}$ - тензорная плотность, обратная к $k_{\mu \nu}$ : $k^{\mu \nu} k_{\nu \sigma}=\delta_{\sigma}^{\mu}$. Тем самым равенство нулю следа импульсов (57) автоматически следует из условия единичности определителя плотности $k_{\mu \nu}$ для производящего функционала (58). В выражении (61) учтено соотношение (59).

По сути дела в качестве новой канонической переменной из метрики выделен ее определитель в некоторой степени

$$
\rho=\left|\operatorname{det} g_{\mu \nu}\right|^{1 /(m(n-1))},
$$

как следует из (59). В дальнейшем симметричную тензорную плотность с единичным определителем $k_{\mu \nu}$ мы для краткости также будем называть метрикой.

Переменные (56) при $n=4$ и $m=1 / 2$ рассматривались в работе [12], где, однако, каноничность преобразования отмечена не была.

Прямые вычисления приводят к следующему выражению для скалярной кривизны сечения $x^{0}=$ const в новых координатах:

$$
\widehat{R}=\rho^{-m-2}\left[\rho^{2} R^{(k)}+m(n-2) \rho \partial_{\mu}\left(k^{\mu \nu} \partial_{\nu} \rho\right)+m(n-2)\left(m \frac{n-3}{4}-1\right) k^{\mu \nu} \partial_{\mu} \rho \partial_{\nu} \rho\right] .
$$

"Скалярная кривизна", построенная по метрике $k_{\mu \nu}$, принимает удивительно простой вид

$$
R^{(k)}=\partial_{\mu \nu}^{2} k^{\mu \nu}+\frac{1}{2} k^{\mu \nu} \partial_{\rho} k_{\mu \sigma} \partial_{\nu} k^{\rho \sigma}-\frac{1}{4} k^{\mu \nu} \partial_{\mu} k_{\rho \sigma} \partial_{\nu} k^{\rho \sigma} .
$$

Это выражение не является скаляром относительно преобразований координат $x^{\mu}$, так как $k_{\mu \nu}-$ не тензор, а тензорная плотность. Отметим, что группа диффеоморфизмов сечений $x^{0}=$ const содержит подгруппу, состоящую из преобразований координат $x^{\mu}$, имеющих единичный определитель. Относительно этой подгруппы $k_{\mu \nu}$ является тензором, а $R^{(k)}$ - скаляром.

Из единичности определителя метрики следует, что компоненты обратной метрики $k^{\mu \nu}$ являются полиномами степени $n-2$ по компонентам $k_{\mu \nu}$ :

$$
k^{\mu \nu}=\frac{1}{(n-2) !} \hat{\varepsilon}^{\mu \rho_{1} \ldots \rho_{n-2}} \hat{\varepsilon}^{\nu \sigma_{1} \ldots \sigma_{n-2}} k_{\rho_{1} \sigma_{1}} \ldots k_{\rho_{n-2} \sigma_{n-2}},
$$

где $\left|\hat{\varepsilon}^{\mu_{1} \ldots \mu_{n-1}}\right|=1$ - полностью антисимметричная тензорная плотность ранга $n-1$. Поэтому скалярная кривизна $R^{(k)}$ полиномиальна как по метрике $k_{\mu \nu}$, так и по обратной метрике $k^{\mu \nu}$. 
Динамическая связь в новых переменных принимает вид

$$
\begin{aligned}
H_{\perp}=\rho^{-} & m(n-1) / 2\left[P^{\mu \nu} P_{\mu \nu}-\frac{\rho^{2}}{m^{2}(n-1)(n-2)} P^{2}\right]- \\
& -\rho^{m(n-1) / 2-m-2}\left[\rho^{2} R^{(k)}+m(n-2) \rho \partial_{\mu}\left(k^{\mu \nu} \partial_{\nu} \rho\right)+\right. \\
& \left.+m(n-2)\left(m \frac{n-3}{4}-1\right) k^{\mu \nu} \partial_{\mu} \rho \partial_{\nu} \rho\right]
\end{aligned}
$$

где $P_{\mu \nu}=k_{\mu \rho} k_{\nu \sigma} P^{\rho \sigma}$.

Проанализируем возможность такого выбора постоянной $m$, чтобы динамическая связь имела полиномиальный вид. Оба выражения в квадратных скобках полиномиальны по всем динамическим переменным. Поскольку $n \geqslant 3$, то для положительности степени плотности $\rho$ перед первой квадратной скобкой необходимо выполнение неравенства $m<0$. В этом случае степень $\rho$ перед второй квадратной скобкой будет отрицательна. Таким образом, за счет выбора степени $m$ добиться полиномиальности самой связи $H_{\perp}$ нельзя. Однако связь можно умножить целиком на произвольный множитель, отличный от нуля. При этом поверхность в фазовом пространстве, определяемая данной связью, не изменится. Степень $\rho$, на которую необходимо умножить $H_{\perp}$, будет минимальна тогда, когда степени $\rho$ перед квадратными скобками будут равны. Отсюда следует равенство

$$
m=\frac{2}{n-2} .
$$

Тогда, умножив динамическую связь

$$
K_{\perp}=\rho^{(n-1) /(n-2)} H_{\perp}=\hat{e} H_{\perp},
$$

получим эквивалентную полиномиальную связь

$$
K_{\perp}=P^{\mu \nu} P_{\mu \nu}-\frac{n-2}{4(n-1)} \rho^{2} P^{2}-\rho^{2} \check{R}=0,
$$

где мы ввели скалярную плотность кривизны

$$
\check{R}(\rho, k)=R^{(k)}+2 \frac{\partial_{\mu}\left(k^{\mu \nu} \partial_{\nu} \rho\right)}{\rho}-\frac{n-1}{n-2} \frac{k^{\mu \nu} \partial_{\mu} \rho \partial_{\nu} \rho}{\rho^{2}}, \quad \operatorname{deg} \check{R}=-\frac{2}{n-1} .
$$

Заметим, что "скалярная" кривизна $R^{(k)}$, построенная по метрической плотности $k_{\mu \nu}$, не является тензорной плотностью. Поэтому использование $\check{R}$ вместо $R^{(k)}$ упрощает многие формулы и вычисления.

Умножение динамической связи $H_{\perp}$ на отличный от нуля множитель приводит к изменению множителя Лагранжа (функции хода) на обратный множитель

$$
N \rightarrow \widetilde{N}=N \rho^{-(n-1) /(n-2)} .
$$

В свою очередь, изменение множителей Лагранжа в ОТО эквивалентно преобразованию координат, т.е. не меняет физического содержания теории. 
Кинематические связи в новых динамических переменных сохраняют свою полиномиальность:

$$
H_{\mu}=-2 \nabla_{\nu}\left(P_{\mu}^{\nu}\right)-\frac{n-2}{n-1} \nabla_{\mu}(P \rho)=0
$$

где $\nabla_{\mu}-$ ковариантная производная, построенная по метрике

$$
g_{\mu \nu}=\rho^{2 /(n-2)} k_{\mu \nu},
$$

а опускание индексов производится с помощью тензорной плотности: $P_{\mu}^{\nu}=P^{\nu \rho} k_{\rho \mu}$. Легко проверить, что

$$
\nabla_{\mu} \rho=0, \quad \nabla_{\mu} k_{\nu \rho}=0
$$

Поэтому опускание и подъем индексов с помощью плотности метрики $k_{\mu \nu}$ перестановочны с операцией ковариантного дифференцирования.

Напомним, что в наших обозначениях ковариантная производная от тензорной плотности $\phi$ степени $\operatorname{deg} \phi=r$ определяется выражением

$$
\nabla_{\mu} \phi=\partial_{\mu} \phi+r \Gamma_{\mu} \phi, \quad \Gamma_{\mu}=\Gamma_{\nu \mu}^{\nu}=\frac{n-1}{n-2} \frac{\partial_{\mu} \rho}{\rho} .
$$

Все новые канонические переменные являются тензорными плотностями степеней

$$
\begin{aligned}
\operatorname{deg} k_{\mu \nu} & =\frac{2}{n-1}, & \operatorname{deg} \rho & =-\frac{n-2}{n-1}, \\
\operatorname{deg} P^{\mu \nu} & =-\frac{n+1}{n-1}, & \operatorname{deg} P & =\frac{1}{n-1},
\end{aligned}
$$

что необходимо учитывать при ковариантном дифференцировании.

Исходя из явного выражения для новых канонических переменных (59)-(61), вычислим основные скобки Пуассона. Отличными от нуля являются только три скобки:

$$
\begin{aligned}
{\left[\rho, P^{\prime}\right] } & =\delta \\
{\left[k_{\mu \nu}, P^{\prime \rho \sigma}\right] } & =\left(\delta_{\mu \nu}^{\rho \sigma}-\frac{1}{n-1} k_{\mu \nu} k^{\rho \sigma}\right) \delta, \\
{\left[P^{\mu \nu}, P^{\prime \rho \sigma}\right] } & =\frac{1}{n-1}\left(P^{\mu \nu} k^{\rho \sigma}-P^{\rho \sigma} k^{\mu \nu}\right) \delta .
\end{aligned}
$$

Скобки Пуассона (69) и (70) не имеют канонического вида для фазовых переменных. Это связано с тем, что на поля $k_{\mu \nu}$ и $P^{\mu \nu}$ наложены дополнительные условия (57).

Поскольку гамильтониан в новых канонических переменных

$$
H=\int d \mathbf{x}\left(\tilde{N} K_{\perp}+N^{\mu} H_{\mu}\right)
$$

полиномиален, то уравнения движения также полиномиальны. Прямые вычисления с использованием скобок Пуассона (68)-(70) приводят к следующим уравнениям 
движения:

$$
\begin{aligned}
\dot{\rho}= & -\frac{n-2}{2(n-1)} \tilde{N} \rho^{2} P+\frac{n-2}{n-1} \nabla_{\mu} N^{\mu} \rho, \\
\dot{P}= & \frac{n-2}{2(n-1)} \tilde{N} \rho P^{2}+2 \widetilde{N} \rho \check{R}+2 k^{\mu \nu} \nabla_{\mu} \nabla_{\nu} \tilde{N} \rho+\frac{1}{n-1} \nabla_{\mu} N^{\mu} P+N^{\mu} \nabla_{\mu} P, \\
\dot{k}_{\mu \nu}= & 2 \widetilde{N} P_{\mu \nu}+\nabla_{\mu} N_{\nu}+\nabla_{\nu} N_{\mu}-\frac{2}{n-1} \nabla_{\rho} N^{\rho} k_{\mu \nu}, \\
\dot{P}^{\mu \nu}= & -2 \widetilde{N} P^{\mu \rho} P_{\rho}^{\nu}-\rho^{2}\left(\tilde{N} k^{\mu \rho} k^{\nu \sigma} \widehat{R}_{\rho \sigma}-\frac{1}{n-1} \tilde{N} \check{R} k^{\mu \nu}+\nabla^{\mu} \nabla^{\nu} \widetilde{N}-\frac{1}{n-1} \square \tilde{N} k^{\mu \nu}\right)+ \\
& \quad+\nabla_{\rho}\left(N^{\rho} P^{\mu \nu}\right)+\frac{2}{n-1} \nabla_{\rho} N^{\rho} P^{\mu \nu}-\nabla_{\rho} N^{\nu} P^{\mu \rho}-\nabla_{\rho} N^{\mu} P^{\nu \rho},
\end{aligned}
$$

где

$$
N_{\mu}=N^{\nu} k_{\mu \nu}, \quad \nabla^{\mu}=k^{\mu \nu} \nabla_{\nu}, \quad \square=k^{\mu \nu} \nabla_{\mu} \nabla_{\nu}
$$

И

$$
\begin{gathered}
\widehat{R}_{\mu \nu}=R_{\mu \nu}^{(k)}+\frac{n-3}{n-2} \frac{\partial_{\mu \nu}^{2} \rho}{\rho}-\frac{(n-1)(n-3)}{(n-2)^{2}} \frac{\partial_{\mu} \rho \partial_{\nu} \rho}{\rho^{2}}-\frac{n-3}{n-2} \Gamma_{\mu \nu}^{(k) \sigma} \frac{\partial_{\sigma} \rho}{\rho}- \\
-\frac{1}{(n-2)^{2}} k^{\mu \nu} \frac{k^{\sigma \lambda} \partial_{\sigma} \rho \partial_{\lambda} \rho}{\rho^{2}}+\frac{1}{n-2} k_{\mu \nu} \frac{\partial_{\sigma}\left(k^{\sigma \lambda} \partial_{\lambda} \rho\right)}{\rho} .
\end{gathered}
$$

Рассмотрим алгебру связей. Поскольку вместо динамической связи $H_{\perp}$ мы ввели новую связь $K_{\perp}$, то алгебра связей изменится. Несложные вычисления дают

$$
\begin{aligned}
{\left[K_{\perp}, K_{\perp}^{\prime}\right] } & =-\left(\rho^{2} H_{\mu} k^{\mu \nu}+\rho^{\prime 2} H_{\mu}^{\prime} k^{\prime \mu \nu}\right) \delta_{\nu}, \\
{\left[K_{\perp}, H_{\mu}^{\prime}\right] } & =-\left(K_{\perp}+K_{\perp}^{\prime}\right) \delta_{\mu}, \\
{\left[H_{\mu}, H_{\nu}^{\prime}\right] } & =-H_{\nu} \delta_{\mu}-H_{\mu}^{\prime} \delta_{\nu} .
\end{aligned}
$$

По сравнению с исходной алгеброй (53)-(54) изменения касаются скобок Пуассона (71) и (72). Вторая скобка Пуассона (72) носит кинематический характер и определяется тем, что новая связь является не скалярной функцией, а скалярной плотностью степени $\operatorname{deg} K_{\perp}=-2$. Скобка Пуассона (71) является результатом прямого вычисления, которое в новых переменных существенно проще, чем в исходных.

До сих пор мы рассматривали плотность метрики $k_{\mu \nu}$ и сопряженные ей импульсы $P^{\mu \nu}$ вместе с дополнительными условиями (57). Это возможно в классической теории, однако в квантовой теории гравитации возникают проблемы. В функциональном интеграле, который будет рассмотрен в следующем разделе, интегрирование ведется по всем значениям $k_{\mu \nu}$ и $P^{\mu \nu}$. В принципе можно решить связи явно, однако этот путь не представляет интереса, так как полиномиальность при этом будет утеряна. Поэтому мы остановимся на описании многообразия $\mathbb{N}$, задаваемого координатами $k_{\mu \nu}$ и $P^{\mu \nu}$, подробно. Для простоты мы предположим, что координаты пробегают все возможные вещественные значения и, следовательно, многообразие $\mathbb{N}$ топологически тривиально и диффеоморфно евклидову пространству $\mathbb{R}^{n(n-1)}$. На этом многообразии заданы скобки Пуассона координат между собой (69), (70) и, следовательно, определена пуассонова структура. Нетрудно проверить, что эта 
структура является вырожденной. Это значит, что все многообразие $\mathbb{N}$ является не симплектическим, а только пуассоновым многообразием (см., например, [16]). Поскольку ранг пуассоновой структуры равен $n(n-1)-2$, то на пуассоновом многообразии $\mathbb{N}$ существуют две функционально независимые функции Казимира:

$$
C_{1}=\operatorname{det} k_{\mu \nu}, \quad C_{2}=\frac{1}{n-1} P^{\mu \nu} k_{\mu \nu}
$$

(введение постоянного множителя $1 /(n-1)$ в $C_{2}$ упростит в дальнейшем ряд формул). Действительно, из определения пуассоновой структуры (69), (70) следует, что скобки Пуассона функций $C_{1}$ и $C_{2}$ со всеми координатами равны нулю:

$$
\left[C_{1,2}, k_{\mu \nu}^{\prime}\right]=\left[C_{1,2}, P^{\prime \mu \nu}\right]=\left[C_{1,2}, \rho^{\prime}\right]=\left[C_{1,2}, P^{\prime}\right]=0 .
$$

Отсюда вытекает, что скобки Пуассона этих функций с произвольной дифференцируемой функцией $f \in \mathcal{C}^{1}(\mathbb{N})$ равны нулю, $\left[C_{1,2}, f^{\prime}\right]=0$, и, следовательно, функции $C_{1}$ и $C_{2}$ являются функциями Казимира. Пуассонова структура, ограниченная на сечения $\mathbb{V} \subset \mathbb{N}$, которые определяются уравнениями $C_{1,2}=$ const, невырожденна. Следовательно, эти сечения являются симплектическими.

На пуассоновом многообразии $\mathbb{N}$ локально всегда можно выбрать координаты, связанные с симплектическими сечениями $C_{1,2}=$ const. Обозначим координаты на этих сечениях через $\left(q_{\mathrm{A}}, p^{\mathrm{A}}\right), \mathrm{A}, \mathrm{B}, \ldots=1, \ldots, n(n-1) / 2-1$. Выберем координаты $q_{\mathrm{A}}$ и $p^{\mathrm{A}}$ таким образом, чтобы было справедливо представление

$$
k_{\mu \nu}=\left|C_{1}\right|^{1 /(n-1)} \stackrel{\circ}{k}_{\mu \nu}, \quad P^{\mu \nu}=\stackrel{\circ}{P}^{\mu \nu}+C_{2} k^{\mu \nu},
$$

где элементы матриц $\stackrel{\circ}{k}_{\mu \nu}(q)$ и $\stackrel{\circ}{P}^{\mu \nu}(p)$ зависят соответственно только от $q_{\mathrm{A}}$ и $p^{\mathrm{A}}$. Очевидно, что такие представления всегда существуют. В качестве недостающих координат на $\mathbb{N}$ выберем сами функции Казимира. Таким образом, мы имеем локальную систему координат

$$
\left(k_{\mu \nu}, P^{\mu \nu}\right) \leftrightarrow\left(q_{\mathrm{A}}, C_{1}\right),\left(p^{\mathrm{A}}, C_{2}\right)
$$

В новых координатах связи (57) имеют простой вид

$$
C_{1}= \pm 1, \quad C_{2}=0
$$

Для $C_{1}$ выбирается знак “+” или “-”, если пространство имеет соответственно четную или нечетную размерность.

Посмотрим на каноническое преобразование (59)-(61) с другой точки зрения. Строго говоря, каноническое преобразование, рассмотренное в настоящем разделе, является каноническим только между координатами

$$
\left(g_{\mu \nu}, p^{\mu \nu}\right) \leftrightarrow(\rho, P),\left(q_{\mathrm{A}}, p^{\mathrm{A}}\right)
$$

Новым фазовым пространством ОТО в рассматриваемом случае является многообразие $\mathbb{R}_{+} \times \mathbb{R} \times \mathbb{V}$, где $\rho \in \mathbb{R}_{+}, \quad P \in \mathbb{R}$, и подмногообразие $\mathbb{V} \subset \mathbb{N}$ определяется 
двумя фиксированными значениями функций Казимира (77). По построению скобки Пуассона координат на $\mathbb{V}$ имеют канонический вид:

$$
\left[q_{\mathrm{A}}, p^{\mathrm{B}}\right]=\delta_{\mathrm{A}}^{\mathrm{B}}, \quad\left[q_{\mathrm{A}}, q_{\mathrm{B}}\right]=0, \quad\left[p^{\mathrm{A}}, p^{\mathrm{B}}\right]=0 .
$$

Полиномиальность связей достигнута за счет расширения пространства $\mathbb{V}$ до пуассонова многообразия $\mathbb{N}$ со скобками Пуассона (68)-(70). Если решить дополнительные связи (57) явно, то полиномиальность будет утеряна. В этом нет ничего необычного. Например, электродинамика содержит связи, явное решение которых приводит даже к нелокальному действию для физических степеней свободы (см., например, [17]).

Скобки Пуассона функций Казимира между собой равны нулю $\left[C_{1}, C_{2}\right]=0$. C точки зрения гамильтонова формализма их можно было бы отнести к связям первого рода, генерирующим калибровочные преобразования. Однако эти преобразования тривиальны, так как скобки Пуассона функций Казимира со всеми координатами фазового пространства равны нулю. Такое возможно только на пуассоновом многообразии с вырожденной пуассоновой структурой. На симплектическом многообразии функций Казимира не существует.

Пуассоново многообразие $\mathbb{N}$ можно оснастить второй на этот раз канонической скобкой Пуассона. Относительно новой канонической структуры подмногообразие $\mathbb{V} \subset \mathbb{N}$ задается двумя связями второго рода (77). Тогда прежняя вырожденная пуассонова структура (69), (70) будет являться не чем иным, как скобкой Дирака по отношению к канонической пуассоновой структуре на $V$.

Отметим еще одно обстоятельство. В гравитации мы предполагаем, что метрика пространства-времени $g_{\alpha \beta}$ имеет лоренцеву сигнатуру и, следовательно, невырожденна. В квантовой теории гравитации интегрирование в функциональном интеграле ведется по всем независимым компонентам метрики, и учесть это обстоятельство можно только за счет ограничения области интегрирования. При расширении фазового пространства до пуассонова многообразия область интегрирования расширяется до евклидова пространства. При этом невырожденность метрики на физическом подпространстве автоматически обеспечивается наличием $\delta$-функций в подынтегральном выражении.

\section{8. ПРОИЗВОДЯЩИЙ ФУНКЦИОНАЛ ДЛЯ ФУНКЦИЙ ГРИНА}

Подытожим результаты вычислений в наиболее интересном случае четырехмерного пространства-времени и выпишем в явном виде производящий функционал для функций Грина.

Полиномиальная гамильтонова формулировка ОТО в предыдущем разделе дана на пуассоновом многообразии $\mathbb{R}_{+} \times \mathbb{R} \times \mathbb{N}$ с координатами $(\rho, P) \in \mathbb{R}_{+} \times \mathbb{R}$ и $\left(k_{\mu \nu}, P^{\mu \nu}\right) \in \mathbb{N}$. Размерность этого многообразия при $n=4$ равна 14 . Пуассонова структура определена отличными от нуля скобками Пуассона

$$
\begin{aligned}
{\left[\rho, P^{\prime}\right] } & =\delta \\
{\left[k_{\mu \nu}, P^{\prime \rho \sigma}\right] } & =\left(\delta_{\mu \nu}^{\rho \sigma}-\frac{1}{3} k_{\mu \nu} k^{\rho \sigma}\right) \delta, \\
{\left[P^{\mu \nu}, P^{\prime \rho \sigma}\right] } & =\frac{1}{3}\left(P^{\mu \nu} k^{\rho \sigma}-P^{\rho \sigma} k^{\mu \nu}\right) \delta .
\end{aligned}
$$


Она вырожденна и имеет ранг 12, что совпадает с размерностью фазового пространства ОТО. На $\mathbb{N}$ существуют две функции Казимира (74). Сечение $C_{1}=-1$, $C_{2}=0$ является симплектическим подмногообразием $\mathbb{V} \in \mathbb{N}$ и определяет фазовое пространство ОТО.

В новых переменных действие ОТО имеет вид

$$
S_{\mathrm{HE}}=\int d x\left(P \dot{\rho}+P^{\mu \nu} \dot{k}_{\mu \nu}-H-\partial_{\mu} B^{\mu}\right),
$$

где гамильтонова плотность $H$ равна линейной комбинации связей

$$
H=\tilde{N} K_{\perp}+N^{\mu} H_{\mu}
$$

и $\widetilde{N}$ и $N^{\mu}$ - множители Лагранжа. К действию (79) добавлен граничный член $\partial_{\mu} B^{\mu}$ на пространственном сечении $x^{0}=$ const, который записан в виде дивергенции от некоторой функции $B^{\mu}\left(\rho, k_{\mu \nu}, P, P^{\mu \nu}\right)$ канонических переменных. Ниже мы кратко обсудим необходимость добавления этого важного слагаемого к действию, не конкретизируя его вид. Связи

$$
\begin{aligned}
& K_{\perp}=P^{\mu \nu} P_{\mu \nu}-\frac{1}{6} \rho^{2} P^{2}-\rho^{2} R^{(k)}-2 \rho \partial_{\mu}\left(k^{\mu \nu} \partial_{\nu} \rho\right)+\frac{3}{2} k^{\mu \nu} \partial_{\mu} \rho \partial_{\nu} \rho, \\
& H_{\mu}=-2 \partial_{\nu}\left(P^{\nu \sigma} k_{\sigma \mu}\right)+P^{\nu \sigma} \partial_{\mu} k_{\nu \sigma}-\frac{2}{3} \partial_{\mu}(P \rho)+P \partial_{\mu} \rho
\end{aligned}
$$

полиномиальны, являются связями первого рода и удовлетворяют алгебре (71)-(73). Скалярная кривизна $R^{(k)}$ для плотности метрики $k_{\mu \nu}$ с единичным определителем имеет вид (63). Связь $K_{\perp}$ квадратична по импульсам и переменной $\rho$. Она представляет собой полином пятой степени по плотности метрики $k_{\mu \nu}$ (и ее частным производным). Связь $H_{\mu}$ линейна как по импульсам, так и по координатам.

Выражение для производящего функционала функций Грина в виде континуального интеграла по фазовому пространству [13] легко обобщается на пуассоново многообразие. Для простоты введем новое обозначение для вторичных связей и множителей Лагранжа:

$$
\left\{H_{a}\right\}=\left\{K_{\perp}, H_{\mu}\right\}, \quad\left\{N^{a}\right\}=\left\{\tilde{N}, N^{\mu}\right\}, \quad a=0,1,2,3 .
$$

Зафиксируем инвариантность относительно общих преобразований координат с помощью четырех калибровочных условий $F^{a}=0$. Будем считать, что калибровка каноническая, т.е.

$$
\operatorname{det}\left[H_{a}, F^{b}\right] \neq 0 \text {. }
$$

Производящий функционал в канонической форме для функций Грина для метрики с точностью до нормировочной постоянной задается функциональным интегралом [13]

$$
\begin{aligned}
Z(J)=\int & D\left(g_{\mu \nu}\right) D\left(p^{\mu \nu}\right) D\left(N^{a}\right) \exp \left\{\frac{i}{h} \int d x\left(p^{\mu \nu} \dot{g}_{\mu \nu}-N^{a} H_{a}-\partial_{\mu} B^{\mu}+g_{\mu \nu} J^{\mu \nu}\right)\right\} \times \\
& \times \operatorname{det}\left[H_{a}, F^{b}\right] \prod_{a} \delta\left(H_{a}\right) \prod_{a} \delta\left(F^{a}\right),
\end{aligned}
$$


где

$$
D\left(g_{\mu \nu}\right)=\prod_{x, \mu \leqslant \nu} d g_{\mu \nu}, \quad D\left(p^{\mu \nu}\right)=\prod_{x, \mu \leqslant \nu} d p^{\mu \nu}, \quad D\left(N^{a}\right)=\prod_{x, a} d N^{a}
$$

и $J^{\mu \nu}$ - источники для метрики. Поскольку мы записали источники только для пространственной метрики, то $Z(J)$ - производящий функционал только для "координатных" функций Грина [17]. Так как якобиан любого канонического преобразования и в частности преобразования (78) равен единице, то выражение для функционального интеграла можно записать в эквивалентном виде

$$
\begin{aligned}
Z(J)=\int & D(\rho) D(P) D\left(q_{\mathrm{A}}\right) D\left(p^{\mathrm{A}}\right) D\left(N^{a}\right) \times \\
& \times \exp \left\{\frac{i}{h} \int d x\left(P \dot{\rho}+p^{\mathrm{A}} \dot{q}_{\mathrm{A}}-N^{a} H_{a}-\partial_{\mu} B^{\mu}+\rho J+q_{\mathrm{A}} J^{\mathrm{A}}\right)\right\} \times \\
& \times \operatorname{det}\left[H_{a}, F^{b}\right] \prod_{a} \delta\left(H_{a}\right) \prod_{a} \delta\left(F^{a}\right) .
\end{aligned}
$$

В таком виде связи $H_{a}$ неполиномиальны по координатам $q_{\mathrm{A}}$ и импульсам $p^{\mathrm{A}}$. Чтобы привести связи к полиномиальному виду, необходимо расширить интегрирование по всему пуассонову многообразию $\mathbb{N}$. Это можно сделать, введя дополнительные $\delta$-функции:

$$
\begin{aligned}
Z(J)=\int & D(\rho) D(P) D\left(k_{\mu \nu}\right) D\left(P^{\mu \nu}\right) D\left(N^{a}\right) \times \\
& \times \exp \left\{\frac{i}{h} \int d x\left(P \dot{\rho}+P^{\mu \nu} \dot{k}_{\mu \nu}-N^{a} H_{a}-\partial_{\mu} B^{\mu}+\rho J+k_{\mu \nu} \tilde{J}^{\mu \nu}\right)\right\} \times \\
& \times \operatorname{det}\left[H_{a}, F^{b}\right] \delta\left(C_{1}+1\right) \delta\left(C_{2}\right) \prod_{a} \delta\left(H_{a}\right) \prod_{a} \delta\left(F^{a}\right) .
\end{aligned}
$$

Две новые $\delta$-функции снимают интегрирование по дополнительным переменным и ограничивают интегрирование по пуассонову многообразию $\mathbb{N}$ до интегрирования по симплектическому сечению (77). Чтобы выполнить соответствующее интегрирование, необходимо совершить преобразование координат (76).

Для доказательства эквивалентности предложенного выражения для производящего функционала по пуассонову многообразию (83) исходному функциональному интегралу по фазовому пространству (82) необходимо доказать, что якобиан преобразования координат (76)

$$
D\left(q_{\mathrm{A}}\right) D\left(C_{1}\right) D\left(p^{\mathrm{A}}\right) D\left(C_{2}\right)=D\left(k_{\mu \nu}\right) D\left(P^{\mu \nu}\right)\left|\frac{\partial\left(q_{\mathrm{A}}, C_{1}, p^{\mathrm{A}}, C_{2}\right)}{\partial\left(k_{\mu \nu}, P^{\mu \nu}\right)}\right|
$$

равен единице на поверхности связей (77).

Это нетрудно сделать. Пусть координаты $q_{\mathrm{A}}$ параметризуют матрицу $k_{\mu \nu}$ с единичным определителем произвольным образом. Тогда

$$
\left|\frac{\partial\left(q_{\mathrm{A}}, C_{1}, p^{\mathrm{A}}, C_{2}\right)}{\partial\left(k_{\mu \nu}, P^{\mu \nu}\right)}\right|=\left|\frac{\partial\left(q_{\mathrm{A}}, C_{1}\right)}{\partial\left(k_{\mu \nu}\right)}\right|\left|\frac{\partial\left(p^{\mathrm{A}}, C_{2}\right)}{\partial\left(P^{\mu \nu}\right)}\right|,
$$


поскольку матрица

$$
\frac{\partial\left(q_{\mathrm{A}}, C_{1}\right)}{\partial\left(P^{\mu \nu}\right)}=0 .
$$

Из определения обобщенных импульсов (60) следует, что

$$
p^{\mathrm{A}}=-\frac{\delta F}{\delta q_{\mathrm{A}}}=-\frac{\delta F}{\delta k_{\mu \nu}} \frac{\partial k_{\mu \nu}}{\partial q_{\mathrm{A}}}=P^{\mu \nu} \frac{\partial k_{\mu \nu}}{\partial q_{\mathrm{A}}} .
$$

Из представления (75) для метрики $k_{\mu \nu}$ вытекает

$$
k_{\mu \nu}=(n-1) C_{1} \frac{\partial k_{\mu \nu}}{\partial C_{1}} .
$$

Поэтому

$$
\frac{\partial C_{2}}{\partial P^{\mu \nu}}=\frac{1}{n-1} k_{\mu \nu}=C_{1} \frac{\partial k_{\mu \nu}}{\partial C_{1}}
$$

Следовательно,

$$
\frac{\partial\left(p^{\mathrm{A}}, C_{2}\right)}{\partial\left(P^{\mu \nu}\right)}=\frac{\partial k_{\mu \nu}}{\partial\left(q_{\mathrm{A}}, C_{1}\right)} C_{1} .
$$

Отсюда вытекает, что якобиан преобразования координат на поверхности связей равен по модулю единице:

$$
\left|\frac{\partial\left(q_{\mathrm{A}}, C_{1}, p^{\mathrm{A}}, C_{2}\right)}{\partial\left(k_{\mu \nu}, P^{\mu \nu}\right)}\right|_{C_{1}=-1}=1 .
$$

Этим обстоятельством объясняется введение численного множителя в функцию Казимира $C_{2}(74)$. В противном случае якобиан преобразования координат был бы равен некоторой ненулевой постоянной, которую, впрочем, можно включить в определение нормировочного множителя производящего функционала.

Обсудим включение граничного слагаемого $\partial_{\mu} B^{\mu}$ в действие ОТО. Исходное выражение для производящего функционала (82) оправданно тем, что после решения всех связей и калибровочных условий возникает функциональный интеграл только по физическим степеням свободы с мерой, которая равна единице. В этом случае гамильтониан на поверхности связей обращается в нуль: $N^{a} H_{a}=0$. С другой стороны, мы знаем, что динамика физических степеней свободы нетривиальна. Возможный выход из возникшего противоречия был предложен в работе [2]. Если калибровочное условие явно зависит от времени, то нетривиальный гамильтониан для физических степеней свободы на поверхности связей возникает из кинетического члена $p^{\mu \nu} \dot{g}_{\mu \nu}$. Однако это не единственное возможное решение проблемы. В книге [17] в действие был добавлен граничный член для получения нетривиального гамильтониана для физических степеней свободы в канонической калибровке, не зависящей от времени явно. Важность граничного слагаемого в ОТО в настоящее время общепризнанна, однако его роль во многих случаях остается неясной из-за значительных технических трудностей. В двумерной гравитации, где связи решаются явно без каких-либо приближений, доказано [18], что для калибровочных условий, не зависящих явно от времени, гамильтониан для физических степеней 
свободы возникает из граничного слагаемого. А именно, слагаемое $\partial_{\mu} B^{\mu}$ на поверхности связей равно гамильтоновой плотности для физических степеней свободы и не имеет вида дивергенции от какой-либо функции. Это утверждение локально и не зависит от того, замкнута вселенная или нет.

Отметим еще одно важное обстоятельство. Для асимптотически плоского пространства-времени объемный интеграл от $\partial_{\mu} B^{\mu}$ равен поверхностному интегралу от $B^{\mu}$ и совпадает с массой для решения Шварцшильда. Поэтому этот поверхностный интеграл был предложен в качестве определения полной энергии гравитационного поля [2]. При этом считалось, что полная энергия замкнутой вселенной равна нулю, так как граница у нее отсутствует и, следовательно, никакого поверхностного интеграла не возникает. Это так, если связи допускают в общем случае гладкие решения для нефизических степеней свободы на компактных многообразиях. Пример двумерной гравитации (который включает в себя сферически-симметричные решения OTO) показывает, что в случае общего положения связи не имеют непрерывных решений на окружности. В таком случае для постановки вариационной задачи для компактного пространства на нем необходимо сделать разрез и добавить граничное слагаемое. Это приводит к нетривиальному выражению для полной энергии замкнутых вселенных.

Можно сказать по-другому. Дивергенция $\partial_{\mu} B^{\mu}$ на поверхности связей, будучи выражена через физические степени свободы, не имеет вида дивергенции от какойлибо функции. Эта гамильтонова плотность для физических степеней свободы имеет один и тот же вид независимо от того, является ли вселенная замкнутой или открытой. Поэтому это выражение можно принять в качестве определения плотности энергии гравитационного поля. Разумеется, такое определение нековариантно и зависит от выбора системы координат.

\section{9. ОДНОРОДНАЯ И ИЗОТРОПНАЯ ВСЕЛЕННАЯ}

Для того чтобы продемонстрировать новые переменные, которые были введены в разделе 7, рассмотрим фридмановскую вселенную [19]. Сначала мы напомним вывод уравнений в лагранжевом формализме. Затем для сравнения переформулируем модель на гамильтоновом языке в старых и новых переменных, в которых гамильтониан принимает полиномиальный вид.

В модели вселенной, которая была предложена Фридманом и является основой для большинства современных космологических моделей, предполагается, что пространство в каждый момент времени (все сечения $x^{0}=t=$ const) является римановым многообразием постоянной кривизны. Сделанное предположение соответствует однородной и изотропной вселенной. Этому условию удовлетворяет метрика

$$
d s^{2}=d t^{2}+a^{2} \stackrel{\circ}{g}_{\mu \nu} d x^{\mu} d x^{\nu}
$$

где $a=a(t)$ - масштабный фактор, зависящий только от времени, а $\stackrel{\circ}{g}_{\mu \nu}-$ (отрицательно определенная) метрика пространства постоянной кривизны, которая по предположению не зависит от времени. Конкретный вид метрики пространства 
постоянной кривизны $\stackrel{\circ}{g}_{\mu \nu}$ зависит от системы координат и для дальнейшего рассмотрения не важен. Для простоты мы ограничимся наиболее важным случаем четырехмерного пространства-времени $(n=4)$.

Метрика пространства-времени удовлетворяет уравнениям Эйнштейна

$$
R_{\alpha \beta}-\frac{1}{2} R g_{\alpha \beta}+\frac{1}{2} \Lambda g_{\alpha \beta}=-\frac{1}{2} T_{\alpha \beta},
$$

где мы ввели космологическую постоянную $\Lambda$ и тензор энергии-импульса материи $T_{\alpha \beta}$. Тензор энергии-импульса материи в сопутствующей системе координат имеет вид [20]

$$
T^{\alpha}{ }_{\beta}=\left(\begin{array}{cccc}
\mathcal{E} & 0 & 0 & 0 \\
0 & -\mathcal{P} & 0 & 0 \\
0 & 0 & -\mathcal{P} & 0 \\
0 & 0 & 0 & -\mathcal{P}
\end{array}\right),
$$

где $\mathcal{E}$ и $\mathcal{P}$ - плотность энергии и давление материи, соответственно. Для однородной и изотропной вселенной в выбранной системе координат эти плотности зависят только от времени: $\mathcal{E}=\mathcal{E}(t)$ и $\mathcal{P}=\mathcal{P}(t)$.

Мы предполагаем также, что уравнение состояния материи задается некоторой функцией плотности энергии от давления

$$
\mathcal{E}=\mathcal{E}(\mathcal{P})
$$

Поскольку тензор энергии-импульса (87) не был получен вариацией некоторого инвариантного действия для полей материи по метрике, то плотность энергии $\mathcal{E}$ не может быть произвольной функцией. Действительно, из тождеств Бианки следует, что ковариантная дивергенция от левой части уравнений Эйнштейна тождественно равна нулю. Поэтому из уравнений Эйнштейна следует уравнение для тензора энергии-импульса:

$$
\nabla_{\alpha} T_{\beta}^{\alpha}=0 .
$$

Для метрики (85) и тензора энергии-импульса (87) эти четыре соотношения сводятся к одному нетривиальному уравнению:

$$
\dot{\mathcal{E}}+\frac{3 \dot{a}}{a}(\mathcal{E}+\mathcal{P})=0
$$

При заданном уравнении состояния (88) мы имеем одно дифференциальное уравнение первого порядка, которое перепишем в виде

$$
\frac{d \mathcal{E}}{\mathcal{E}+\mathcal{P}(\mathcal{E})}=-3 \frac{d a}{a} .
$$

Решение этого уравнения задает плотность энергии $\mathcal{E}$ как функцию от масштабного фактора $a$.

Поскольку метрика (85) должна удовлетворять уравнениям Эйнштейна (86), то это приводит к уравнениям для масштабного фактора. Несложные вычисления 
дают следующие выражения для тензора Эйнштейна:

$$
\begin{gathered}
R_{0}{ }^{0}-\frac{1}{2} R=3 \frac{K_{0}-\dot{a}^{2}}{a^{2}}, \\
R_{0}{ }^{\mu}=0, \\
R_{\mu}{ }^{\nu}-\frac{1}{2} R \delta_{\mu}^{\nu}=-\frac{1}{a^{2}}\left(2 a \ddot{a}+\dot{a}^{2}-K_{0}\right) \delta_{\mu}^{\nu},
\end{gathered}
$$

где в наших обозначениях $K_{0}$ - скалярная кривизна трехмерной сферы $\left(K_{0}=1\right)$, евклидова пространства $\left(K_{0}=0\right)$ или однополостного гиперболоида $\left(K_{0}=-1\right)$. Теперь нетрудно увидеть, что из уравнений Эйнштейна следует только два нетривиальных уравнения для масштабного фактора:

$$
\begin{gathered}
3 \frac{K_{0}-\dot{a}^{2}}{a^{2}}+\Lambda+\frac{1}{2} \mathcal{E}=0, \\
-\frac{1}{a^{2}}\left(2 a \ddot{a}+\dot{a}^{2}-K_{0}\right)+\Lambda-\frac{1}{2} \mathcal{P}=0 .
\end{gathered}
$$

Поскольку уравнения Эйнштейна с учетом (89) линейно зависимы, то, как нетрудно проверить, уравнение (92) является следствием (91) и (89). Поэтому второе уравнение (92) можно опустить. Мы этого делать не будем, так как оно понадобится для канонической формулировки модели фридмановской вселенной.

Таким образом, при заданном уравнении состояния (88), решая уравнение (90), мы находим зависимость плотности энергии от масштабного фактора $\mathcal{E}=\mathcal{E}(a)$. Подставляя эту функцию в уравнение (91), мы получаем обыкновенное дифференциальное уравнение первого порядка для масштабного фактора. Это и есть основное уравнение в стандартных моделях однородной и изотропной вселенной.

Перейдем к канонической формулировке. Для метрики (85) имеем

$$
N=1, \quad N_{\mu}=0, \quad g_{\mu \nu}=a^{2} \stackrel{\circ}{g}_{\mu \nu} .
$$

Тензор внешней кривизны (32) и элемент объема равны

$$
\begin{gathered}
K_{\mu \nu}=-a \dot{a} \stackrel{\circ}{g}_{\mu \nu}, \quad K=-3 \frac{\dot{a}}{a}, \\
\hat{e}=a^{3} \stackrel{\circ}{e}, \quad \stackrel{\circ}{e}=\sqrt{\left|\operatorname{det} \stackrel{\circ}{g}_{\mu \nu}\right|} .
\end{gathered}
$$

Канонические импульсы (44), сопряженные пространственной метрике, принимают вид

$$
p^{\mu \nu}=-2 \dot{a}^{\circ} e^{\mu \nu} g^{\mu \nu} .
$$

Для этих импульсов бесследовая часть тождественно равна нулю:

$$
\tilde{p}^{\mu \nu}=0, \quad p=-6 a^{2} \dot{a} \stackrel{\circ}{ } .
$$

Поскольку функция сдвига для вселенной Фридмана равна нулю, то гамильтонова плотность определяется только динамической связью (48):

$$
H_{\perp}=-6 \stackrel{\circ}{e}\left[\left(\dot{a}^{2}-K_{0}\right) a-\frac{1}{3} \Lambda a^{3}-\frac{1}{6} \mathcal{E} a^{3}\right],
$$


где мы учли вклады от космологической постоянной и полей материи. В это выражение мы не можем подставить след импульсов $p$ вместо производной по времени $\dot{a}$, так как он не является переменной, канонически сопряженной к масштабному фактору.

Чтобы найти импульс, сопряженный к $a$, перепишем лагранжиан (42) для метрики (85):

$$
\mathcal{L}_{\mathrm{ADM}}=-6 \stackrel{\circ}{ }\left[\left(\dot{a}^{2}+K_{0}\right) a+\frac{1}{3} \Lambda a^{3}+\frac{1}{6} \mathcal{E} a^{3}\right] .
$$

Поскольку вся зависимость от пространственных координат содержится в элементе объема $\stackrel{\circ}{e}$, то это выражение можно проинтегрировать по пространству. Опуская постоянный множитель $-6 V$, где $V$ - объем пространства (бесконечный для евклидова пространства и однополостного гиперболоида), получаем лагранжиан для масштабного фактора

$$
L=\left(\dot{a}^{2}+K_{0}\right) a+\frac{1}{3} \Lambda a^{3}+\frac{1}{6} \mathcal{E} a^{3},
$$

который не зависит от пространственных координат. Это обычный лагранжиан точечной частицы, движущейся в одномерном пространстве с координатой $a \in \mathbb{R}_{+}$.

При получении лагранжиана для масштабного фактора мы опустили отрицательный множитель, для того чтобы кинетический член $a \dot{a}^{2}$ имел положительный знак. Тем самым мы изменили общий знак перед действием и, следовательно, знак перед энергией. Следует помнить, что вклад в энергию кинетического члена для масштабного фактора отрицателен.

Из лагранжиана (95) следует выражение для импульса, сопряженного масштабному фактору:

$$
p_{a}=\frac{\partial L}{\partial \dot{a}}=2 a \dot{a}
$$

которое отличается от следа импульсов (93) множителем и не зависит от пространственных координат. Гамильтониан для масштабного фактора, соответствующий лагранжиану (95), принимает вид

$$
H=\frac{1}{4 a} p_{a}^{2}-K_{0} a-\frac{1}{3} \Lambda a^{3}-\frac{\mathcal{E}}{6} a^{3} .
$$

Отметим, что это выражение для гамильтониана с точностью до множителя $-6 \mathrm{~V}$ совпадает с тем выражением, которое получается после интегрирования динамической связи (94) по пространству. Это замечание нетривиально, потому что, как хорошо известно, при подстановке в лагранжиан специальных выражений для полевых переменных часть уравнений движения может быть потеряна.

Гамильтоновы уравнения для масштабного фактора имеют вид

$$
\begin{aligned}
\dot{a} & =\frac{1}{2 a} p_{a}, \\
\dot{p}_{a} & =\frac{1}{4 a^{2}} p_{a}^{2}+K_{0}+\Lambda a^{2}+\frac{1}{2} \mathcal{P} a^{2} .
\end{aligned}
$$

Здесь при вычислении скобки Пуассона

$$
\left[p_{a}, \mathcal{E}\right]=\left[p_{a}, a\right] \frac{d \mathcal{E}}{d a}=-3 \frac{\mathcal{E}+\mathcal{P}}{a}
$$


мы воспользовались уравнением (90).

Нетрудно проверить, что гамильтоновы уравнения (97) эквивалентны лагранжеву уравнению второго порядка (92), а гамильтониан (96) пропорционален левой части уравнения (91). Таким образом, мы сформулировали уравнения фридмановской вселенной на гамильтоновом языке. В отличие от обычной гамильтоновой динамики частиц, помимо канонических уравнений движения (97) мы имеем дополнительную связь

$$
H\left(a, p_{a}\right)=0 .
$$

Другими словами, мы ищем только те решения уравнений движения, энергия которых равна нулю. Эта задача самосогласованна, так как энергия сохраняется. Таким образом, мы сформулировали уравнения для масштабного фактора (91) и (92) на гамильтоновом языке и доказали, что при подстановке выражения для метрики (85) не в уравнения Эйнштейна, а в действии мы теряем одну связь (98) для масштабного фактора и соответствующего импульса.

Гамильтониан (96) и уравнения движения (97) неполиномиальны по масштабному фактору. Покажем, что происходит с уравнениями при каноническом преобразовании, рассмотренном в разделе 7. Из формул (59)-(61) следуют выражения для канонических переменных

$$
\begin{aligned}
\rho & =a^{2} e^{\circ 2 / 3}, & k_{\mu \nu} & =\eta_{\mu \nu}, \\
P & =-2 \frac{\dot{\rho}}{\sqrt{\rho}}, & P^{\mu \nu} & =0,
\end{aligned}
$$

внешней кривизны

$$
K_{\mu \nu}=-\frac{1}{2} \dot{\rho} \eta_{\mu \nu}, \quad K=-\frac{3 \dot{\rho}}{2 \rho}
$$

и элемента объема

$$
\hat{e}=\rho^{3 / 2}
$$

после канонического преобразования.

Чтобы упростить вычисления, проще всего разделить переменные, выделив из $\rho$ множитель $q(t)$, зависящий только от времени:

$$
\rho\left(t, x^{\mu}\right)=q(t) e^{\circ / 3}
$$

Тогда лагранжеву плотность, как и прежде, можно проинтегрировать по пространству, а гамильтонова формулировка уравнений сведется к переопределению масштабного фактора

$$
q=a^{2} .
$$

Для новой переменной лагранжиан (95) примет вид

$$
L=\frac{1}{4 \sqrt{q}} \dot{q}^{2}+K_{0} \sqrt{q}+\frac{1}{3} \Lambda q^{3 / 2}+\frac{1}{6} \mathcal{E} q^{3 / 2} .
$$

Импульс, сопряженный к новой динамической переменной $q(t)$, равен

$$
p_{q}=\frac{1}{2 \sqrt{q}} \dot{q} .
$$


Соответствующий гамильтониан содержит неполиномиальный множитель

$$
H=\sqrt{q}\left(p_{q}^{2}-K_{0}-\frac{1}{3} \Lambda q-\frac{1}{6} \mathcal{E} q\right) .
$$

При построении полиномиальной гамильтоновой формулировки динамическая связь была умножена на множитель (64). В результате мы получим новую, теперь уже полиномиальную связь или новый гамильтониан

$$
K=q^{3 / 2} H=q^{2}\left(p_{q}^{2}-K_{0}-\frac{1}{3} \Lambda q-\frac{1}{6} \mathcal{E} q\right) .
$$

Чтобы сохранить гамильтонову форму уравнений движения, мы должны также переопределить время $t \rightarrow \tau$, где новый параметр определяется дифференциальным уравнением

$$
\frac{d \tau}{d t}=q^{-3 / 2}=a^{-3}
$$

Переопределение времени соответствует переопределению множителя Лагранжа (66). Уравнения движения для гамильтониана (102) имеют вид

$$
\begin{gathered}
\frac{d q}{d \tau}=2 q^{2} p_{q} \\
\frac{d p_{q}}{d \tau}=q^{2}\left(\frac{1}{3} \Lambda-\frac{1}{12} \mathcal{E}-\frac{1}{4} \mathcal{P}\right)-\frac{2}{q} K
\end{gathered}
$$

где мы опять воспользовались уравнением (90). Второе слагаемое во втором уравнении может быть отброшено, поскольку в модели есть связь

$$
K\left(q, p_{q}\right)=0 .
$$

Таким образом, в новых переменных мы имеем "частицу", описываемую координатой $q(\tau) \in \mathbb{R}_{+}$и импульсом $p_{q}(\tau) \in \mathbb{R}$, с гамильтонианом (102). При этом на канонические переменные наложена связь (103). Гамильтониан и уравнения движения полиномиальны (если плотность энергии $\mathcal{E}(q)$ полиномиальна по $q$ ) и эквивалентны исходным уравнениям Эйнштейна для масштабного фактора (91), (92).

В модели однородной и изотропной вселенной можно пойти еще дальше и убрать из гамильтониана (102) общий множитель $q^{2}$, переопределив время $t \rightarrow \tau^{\prime}$, где

$$
d \tau^{\prime}=q^{-1 / 2} d t
$$

Тогда мы имеем "частицу" с простым гамильтонианом

$$
K^{\prime}=p_{q}^{2}-K_{0}-\frac{1}{3} \Lambda q-\frac{1}{6} \mathcal{E} q
$$

и соответствующими уравнениями движения:

$$
\begin{aligned}
\frac{d q}{d \tau^{\prime}} & =2 p_{q}, \\
\frac{d p_{q}}{d \tau^{\prime}} & =\frac{1}{3} \Lambda-\frac{1}{12} \mathcal{E}-\frac{1}{4} \mathcal{P} .
\end{aligned}
$$


Кривизна пространства $K_{0}$ при этом вообще не дает вклада в уравнения движения. Система уравнений движения решается при условии равенства нулю гамильтониана: $K^{\prime}=0$. Как и в случае исходной системы уравнений (91), (92), гамильтоновы уравнения движения в лагранжевой форме являются следствием уравнения $K^{\prime}=0$, где вместо импульса необходимо подставить его выражение через производную по времени от масштабного фактора (104). И это уравнение эквивалентно уравнению (91).

В случае пылевидной материи $\mathcal{P}=0$ уравнение $(90)$ легко интегрируется:

$$
\mathcal{E}=\frac{M}{a^{3}}, \quad M=\text { const },
$$

и мы имеем безмассовую частицу, движущуюся в потенциале

$$
-\frac{1}{3} \Lambda q-\frac{M}{6 q^{2}} .
$$

Таким образом, введение новых канонических переменных позволяет переформулировать уравнения для фридмановской вселенной в полиномиальной форме. Поскольку уравнение (91) за прошедшие десятилетия было хорошо изучено, то вряд ли предложенная формулировка даст новые результаты в рассматриваемом случае для изучения классических решений. Она может оказаться полезной для построения квантовой модели фридмановской вселенной, однако этот вопрос требует самостоятельного исследования и выходит за рамки настоящей статьи. Рассмотрение в настоящем разделе ставило цель только продемонстрировать, как общий метод работает в простом случае, где все моменты можно проверить простыми вычислениями.

\section{0. ЗАКЛЮЧЕНИЕ}

В настоящей статье показано, что выделение определителя метрики и сопряженного импульса в качестве дополнительных независимых переменных приводит к канонической формулировке ОТО, в которой все связи полиномиальны. Модель формулируется в полиномиальном виде не в фазовом пространстве, а на пуассоновом многообразии, где скобка Пуассона вырожденна. Подчеркнем, что результирующая модель эквивалентна ОТО.

В новых переменных канонические импульсы пропорциональны неприводимым компонентам импульсов в стандартной метрической формулировке. Это обстоятельство существенно упрощает вычисления, в частности вычисление скобки Пуассона для динамической связи.

Предложенная каноническая формулировка ОТО позволяет записать функциональный интеграл на пуассоновом многообразии. Доказано, что этот интеграл эквивалентен функциональному интегралу по фазовому пространству. Преимущество новой записи производящего функционала для функций Грина состоит в том, что действие и все аргументы $\delta$-функций полиномиальны по независимым переменным. Это приводит к тому, что в диаграммной технике присутствует только конечное число вершин. Это, по-видимому, упростит вычисления в квантовой теории гравитации. 
В качестве примера использования новых переменных рассмотрена модель вселенной Фридмана. В этих переменных гамильтониан и уравнения движения для масштабного фактора упрощаются и принимают полиномиальный вид.

Аналогичное преобразование координат в конфигурационном пространстве, приводящее к полиномиальному действию Гильберта-Эйнштейна, было предложено в работе [21] и недавно переоткрыто [22].

Благодарности. Автор выражает искреннюю благодарность И. В. Воловичу за обсуждение статьи. Работа выполнена при поддержке РФФИ (грант № 05-01-00884) и Программы поддержки ведущих научных школ (грант № НШ-6705.2006.1).

\section{Список литературы}

[1] П.А. М. Дирак, “Теория гравитации в гамильтоновой форме”, Новейшие проблемы гравитачии, Под редакцией Д. Иваненко, ИЛ, М., 1961, 139-158.

[2] R. Arnowitt, S. Deser, S. W. Misner, "The dynamics of general relativity", Gravitation: an introduction to current research, ed. L. Witten, John Wiley \& Sons, N.Y.-London, 1962, 227-265; gr-qc/0405109.

[3] T. Regge, C. Teitelboim, Ann. Phys., 88 (1974), 286-318.

[4] B. S. DeWitt, Phys. Rev., 160:5 (1967), 1113-1148.

[5] C. Teitelboim, Ann. Phys., 79 (1973), 542-557.

[6] K. Kuchar, J. Math. Phys., 17:5 (1976), 792-800.

[7] P. A. M. Dirac, "Interacting gravitational and spinor fields", Recent Developments in General Relativity, Pergamon, PNW - Polish Scientific Publishers, Warsaw, Oxford, 1962, 191-200.

[8] J. Schwinger, Phys. Rev., 130:3 (1963), 1253-1258.

[9] S. Deser, C. J. Isham, Phys. Rev. D, 14:10 (1976), 2505-2510.

[10] J. M. Charap, M. Henneaux, J. E. Nelson, Class. Q. Grav., 5:11 (1988), 1405-1414.

[11] A. Ashtekar, Phys. Rev. D, 36:6 (1987), 1587-1602.

[12] R. S. Tate, Class. Q. Grav., 9 (1992), 101-119.

[13] Л. Д. Фаддеев, ТМФ, 1:1 (1969), 3-18.

[14] P. A. M. Dirac, Can. J. Math., 3:1 (1951), 1-23.

[15] Г. Голдстейн, Классическая механика, Наука, М., 1975.

[16] Y. Choquet-Bruhat, C. DeWitt-Morette, Analysis, Manifolds and Physics, North-Holland, Amsterdam, 1989.

[17] Д. М. Гитман, И. В. Тютин, Каноническое квантование полей со связями, Наука, М., 1986.

[18] M. O. Katanaev, Ann. Phys., 296:1 (2002), 1-50.

[19] А.А. Фридман, ЖКурн. Русск. физ.-хим. о-ва, часть физ., 56:1 (1924), 59; УФН, 80:3 (1963), 439-446; 447-452; 93:2 (1967), 280-287.

[20] Л. Д. Ландау, И. М. Лифшиц, Теория поля, Наука, М., 1988.

[21] A. Peres, Nuovo Cimento, 28:4 (1963), 865-867.

[22] M. O. Katanaev, Gen. Rel. Grav., 38 (2006), 1233-1240; gr-qc/0507026.

Поступила в редакцию 27.10 .2005 , после доработки 27.02.2006 